CERN-PPE/92-175

15 October 1992

\title{
PRECISION TESTS OF THE ELECTROWEAK INTERACTION
}

\author{
Luigi ROLANDI \\ PPE Division, CERN, CH-1211 Geneva 23
}

\begin{abstract}
Recent measurements of various charged current and neutral current processes are presented and compared with the prediction of the Standard Model of the Electroweak Interaction. No discrepancy was found in the experiments'precision which is often higher than $0.5 \%$. A Standard Model fit to the data allows an indirect determination of the top mass: $m_{\text {top }}=145 \pm 25 \mathrm{GeV}$.
\end{abstract}

Talk given at the XXVI ICHEP 1992, Dallas, USA 



\title{
PRECISION TESTS OF THE ELECTROWEAK INTERACTION
}

\author{
Luigi ROLANDI \\ Particle Physics Experiments Division \\ CERN
}

CH-1211 Geneva 23

\begin{abstract}
Recent measurements of various charged current and neutral current processes are presented and compared with the prediction of the Standard Model of the Electroweak Interaction. No discrepancy was found in the experiments' precision which is often higher than $0.5 \%$. A Standard Model fit to the data allows an indirect determination of the top mass: $m_{\text {top }}=145 \pm 25 \mathrm{GeV}$.
\end{abstract}

\section{INTRODUCTION}

The Electroweak Standard Model is a renormalizable theory and any experimental quantity can be predicted theoretically in terms of a finite number of parameters. These parameters are known with some precision from a priori determinations. Any additional measurement can be compared with the prediction. If these agree, the measurement can be used to further constrain the input parameters. Non-agreement indicates inconsistency of the theoretical framework and new physics.

This review summarizes the results of some precise experiments that test the Standard Model in the charged current sector and in the neutral current sector. The various experimental results are presented, compared to the prediction and finally used to constrain the unknown parameters of the model.

Many of the results summarized in this review have been submitted to the Conference as preliminary. They may change in the near future when the analyses will be eventually completed.
Charged current lepton universality

Lepton universality predicts that the coupling of the charged current to the three know leptonic doublets are equal. This hypothesis is verified experimentally comparing the decay width of two reactions that are identical on all aspects except the leptonic flavour involved.

Deviation from universality could point, for example, to the existence of a fourth generation neutrino of large mass $(>46 \mathrm{GeV})$ which mixes with one of the known neutrinos[1].

e- $\mu$ universality

The measurement of the branching ratio $R$ of the rare pion decay into positron and neutrino $\left(\pi^{+} \rightarrow e^{+} \nu\right)$ normalized to the normal pion decay $\pi^{+} \rightarrow \mu^{+} \nu$ can be compared with the theoretical calculation $R_{T H}$ thus allowing a test of e- $\mu$ universality.

$$
R=R_{T H}\left(\frac{g_{e}}{g_{\mu}}\right)^{2}
$$

where $g$ is the coupling constant of the lepton to the charged current. The most recent prediction for $R_{T H}[2]$ is $R_{T H}=(1.2345 \pm$ $0.0010) \times 10^{-4}$ where the uncertainty arises 
from uncalculated but bounded pion structure effects.

Two experiments, one performed at TRIUMF[3] and the other at PSI[4], have recently published new precise measurements of $R$. Positive pions are stopped in an active target and the prompt electrons from the $\pi \rightarrow e \nu$ rear decay are separated from the much larger background $\pi \rightarrow \mu \rightarrow e$ absorbing them in a calorimeter and measuring their energy with high resolution. This information is combined with the pulse height measured in the target, which is different in the two reactions due to the additional energy loss of the muon.

The two experiments obtain consistent results and have similar precision:

$$
\begin{gathered}
R=1.2265 \pm 0.0034 \pm 0.0044 \times 10^{-4}[3] \\
R=1.235 \pm 0.003 \pm 0.004 \times 10^{-4}[4]
\end{gathered}
$$

where the first errors are statistical and the second systematic. They are mainly independent and the two results can be averaged and compared with the theoretical prediction using eqn.1 giving:

$$
\frac{g_{e}}{g_{\mu}}=0.9987 \pm 0.0019
$$

in agreement with the hypothesis of e- $\mu$ universality.

\section{$\mu-\tau$ universality}

A test of the $\mu-\tau$ universality is performed comparing the decay widths of the muon and the tau into electron and two neutrinos. Their ratio is predicted to scale with the 5 th power of the mass of the parent lepton:

$$
\left(\frac{g_{\tau}}{g_{\mu}}\right)^{2}=\operatorname{Br}(\tau \rightarrow e \nu \bar{\nu}) \frac{\tau_{\mu}}{\tau_{\tau}}\left(\frac{m_{\tau}}{m_{\mu}}\right)^{5}
$$

where $m$ is the mass of the lepton, $\tau$ its lifetime and $g$ its coupling constant to the charged current. Radiative corrections[5] are at the level of $0.04 \%$ and can be neglected at the present level of precision.

The mass and the lifetime of the muon are known with very small relative errors compared to the corresponding tau quantities and the error on the ratio of the coupling constants is given by:

$$
\Delta\left(\frac{g_{\tau}}{g_{\mu}}\right)=\frac{1}{2}\left[\frac{\Delta \tau_{\tau}}{\tau_{\tau}} \oplus \frac{\Delta B r}{B r} \oplus 5 \frac{\Delta m_{\tau}}{m_{\tau}}\right]
$$

Recent measurements of the mass, lifetime and electronic branching ratio of the $\tau$ allow a more precise determination of the ratio of the coupling constants. Using previous measurements this ratio was determined to be consistent with unity at the $2.3 \sigma$ level[6].

\section{$\tau$ mass measurement}

The mass of the $\tau$ has been measured at BES in a scan near the $\tau^{+} \tau^{-}$production threshold[7]. Candidate events are identified by requiring $e-\mu$ final state. A likelihood function is used to estimate the $\tau$ mass incorporating the $\tau^{+} \tau^{-}$cross section near threshold, the number of candidate events per scan point and the luminosity integrated at each scan point. Fig. 1 shows the convergence of the predicted mass with each consecutive scan point, the collected luminosities and the number of candidate events. Using 7 candidate events and the information that no event is found at the other scan points, the value of the mass is fitted from the energy dependence of the cross section

$$
m_{\tau}=1776.9_{-0.5}^{+0.4} \pm 0.2 \mathrm{MeV} .
$$

The most important systematic error is due to the uncertainty on the selection efficiency. The energy calibration of BES is determined by several scans of the $J / \Psi$ and $\Psi(2 S)$ peaks and contributes to the systematics on $m_{\tau}$ with an error of $\pm 0.09 \mathrm{MeV}$. 

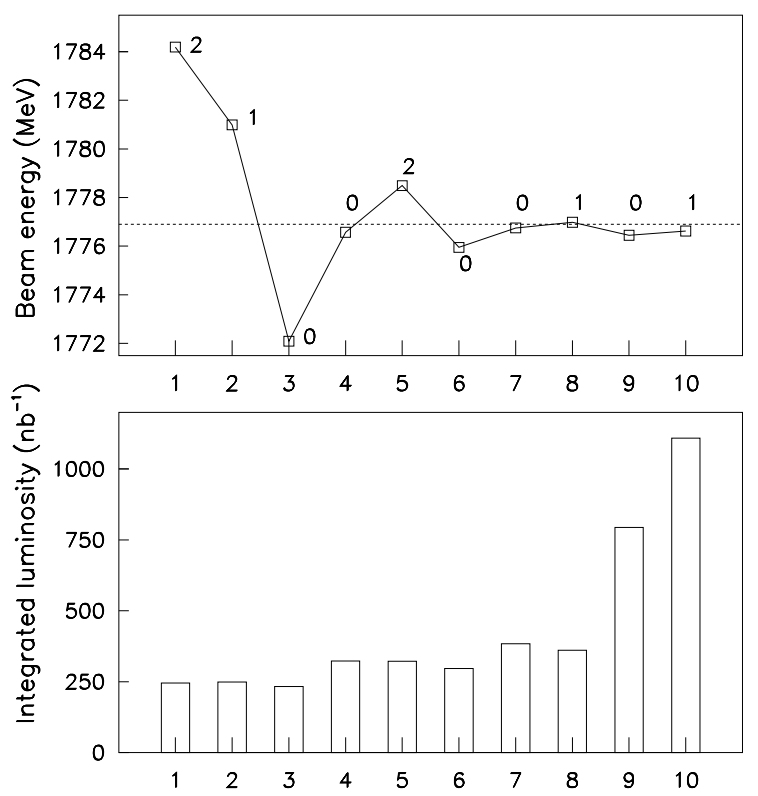

Figure 1. Convergence of the $\tau$ mass with the scan points The number of candidate events at each scan point is also indicated.

This result is $7.2 \mathrm{MeV}$ below the previous world average[8] and reduces the error on the $\tau$ mass by a factor 6 .

Two other independent measurements of the tau mass have been recently performed by Argus[9] $\left(m_{\tau}=1776.3 \pm 2.4 \pm 1.4 \mathrm{MeV}\right)$ and Cleo[10] $\left(m_{\tau}=1777.6 \pm 0.9 \pm 1.6 \mathrm{MeV}\right)$ using clever kinematical constraints.

\section{$\tau$ lifetime measurement}

The lifetime of the $\tau$ has been measured with improved precision at LEP using the silicon microvertex detectors installed recently in ALEPH, DELPHI and OPAL. Typical resolutions on the impact parameter are in the range $10-20 \mu \mathrm{m}$.

The lifetime is measured with various techniques similar to the previous published analysis $[11,12,13,14]$ and also with new methods [15]. In the three-prong decays the secondary vertex is reconstructed allowing a direct measurement of the decay length, while in the oneprong decay the impact parameter technique is used. In the events where both $\tau$ particles decay into one prong the correlations among the impact parameters and the azimuthal angles are used in some analysis[11, 15] to increase the sensitivity.

Fig. 2 shows the new results of the LEP experiments [15] compared with the average of the other experiments $[8,16]$. The precision on the lifetime has been improved by a factor 2 with respect to the previous world average[8].

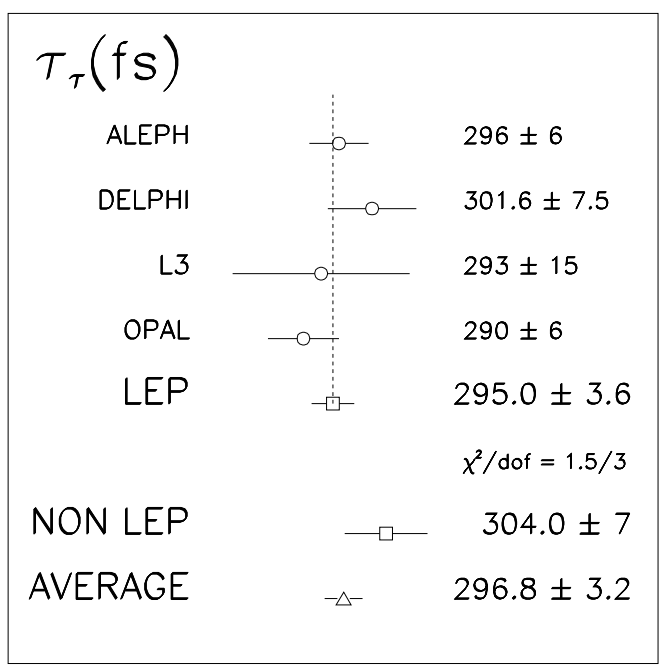

Figure 2. Tau lifetime.

\section{Determination of the $\operatorname{Br} \tau \rightarrow e \nu \bar{\nu}$}

Using its large sample of data, CLEO has measured the electronic branching ratio $B_{e}$ of the $\tau[10]$ selecting events in which both taus decay into electron and normalizing them to the number of $\tau$ pairs expected from the luminosity measurement. Their result is

$$
B_{e}=17.42 \pm 0.15 \pm 0.23 \%
$$

and is as accurate as the previous world average[8].

New measurements have also been reported from LEP[15] on the electronic and muonic branching ratio of the $\tau$ :

$$
\begin{aligned}
& B_{e}=17.68 \pm 0.17 \pm 0.18 \% \\
& B_{\mu}=17.33 \pm 0.17 \pm 0.15 \%
\end{aligned}
$$


Assuming $e-\mu$ universality, the measured $B_{\mu}$ can be used to improve the accuracy on $B_{e} \cdot \bar{B}_{e}$ is obtained as the weighted average between $B_{e}$ and $B_{\mu} / 0.9728^{1}$ :

$$
\begin{array}{ll}
\bar{B}_{e}=17.76 \pm 0.17 \% & \text { LEP } \\
\bar{B}_{e}=17.67 \pm 0.18 \% & \text { NON-LEP } \\
\bar{B}_{e}=17.71 \pm 0.12 \% & \text { AVERAGE }
\end{array}
$$

where the NON-LEP average includes the CLEO measurement and previous experiments[8]. The new measurements improve the error on the electronic branching ratio by a factor 1.6 with respect to the previous world average[8].

\section{$\mu-\tau$ universality, results}

The measurement of $\tau$ mass, lifetime and electronic branching ratio can be now used to perform a test of $\mu-\tau$ universality using eqn.

2.

$$
\begin{aligned}
& 0.992 \pm 0.007 \text { LEP } \\
& \frac{g_{\tau}}{g_{\mu}}=0.974 \pm 0.012 \text { NON LEP } \\
& 0.987 \pm 0.006 \quad \text { ALL DATA }(2.2 \sigma)
\end{aligned}
$$

The measurement of the mass is so precise that it does not contribute to the error (cfr. eqn.3) on the ratio of the coupling constant. The lifetime and the electronic branching ratio contribute with almost equal weight.

The situation is illustrated in fig. 3, where the measured electronic branching ratio is plotted together with the value derived from the lifetime assuming lepton universality and using eqn. 2 with the BES value of the $\tau$ mass.

The direct measurements of the branching ratio are very consistent. The LEP value derived from the lifetime is also very consistent with the direct measurement. The NON LEP determination of the lifetime produces a branching ratio that is $2.2 \sigma$ higher than the direct measurement, but only $1.1 \sigma$ higher

\footnotetext{
${ }^{1} e-\mu$ universality predicts $B_{\mu} / B_{e}=0.9728$ due to the larger $\mu$ mass.
}

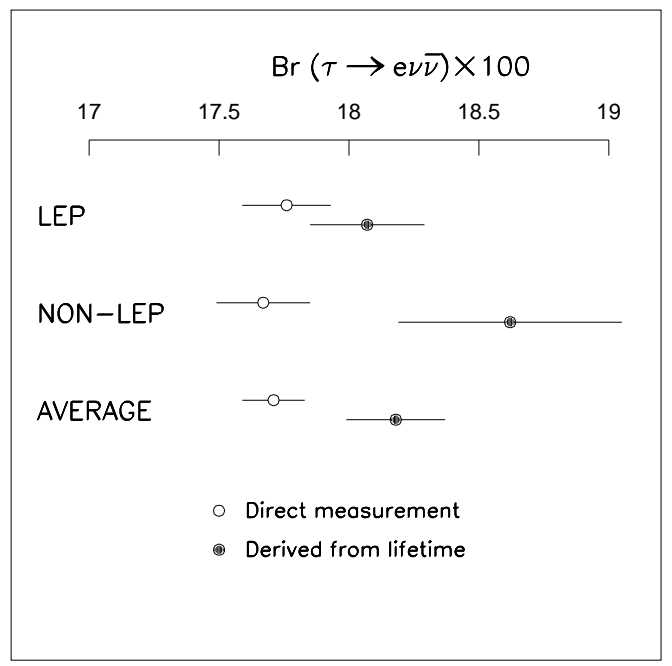

Figure 3. $\operatorname{Br}(\tau \rightarrow e \nu \bar{\nu})$. The direct measurements are compared with those derived from the lifetime, assuming $\mu-\tau$ universality.

than the LEP value derived from the lifetime. When all data are combined, the agreement between the direct measurement of the branching ratio and the derived one remains at $2.2 \sigma$ level.

In conclusion, the new measurements of the $\tau$ parameters allow a test of the $\mu-\tau$ universality with an improvement in precision by more than a factor 2 compared to previous measurements[6]. The ratio of the coupling constants is consistent with 1 at the 2.2 $\sigma$ level.

Neutrino electron scattering

New results on the scattering $\nu e \rightarrow$ ve have been presented by the CHARM II Collaboration[17].

The signature of the process is a single forward scattered electron producing an electromagnetic shower in the calorimeter. The variable $E_{e} \theta_{e}^{2}$ - product of the energy and square of the scattering angle - is kinematically constrained to values smaller than $1 \mathrm{MeV}$ and is used to separate the signal from the background, mainly due to the reaction $\nu A \rightarrow$ $\nu \pi_{0} A$. 
This scattering is a purely leptonic reaction and its interpretation has little theoretical uncertainties. The cross section is small $\left(\sigma_{\text {tot }}^{\nu e} \sim 10^{-4} \sigma_{\text {tot }}^{\text {inclusive }}\right.$ ) resulting in a small number of selected events. In total, about 3000 neutrino electron scattering and 3000 antineutrino electron scattering events have been selected. $\sin ^{2} \theta$ can be extracted directly from the ratio of the two cross sections:

$$
\sin ^{2} \theta_{\nu e}=0.232 \pm 0.006 \pm 0.007 .
$$

From a comparison between the data and the predicted event distribution $d \sigma / d y$ [18], and assuming $g_{a}^{\nu}=1 / 2$, the vector and axialvector coupling constants of the electron to the neutral current are measured at $Q^{2} \sim$ $10^{-2} G e V^{2}$ :

$$
\begin{aligned}
& g_{v}^{e}=-0.025 \pm 0.014 \pm 0.014 \\
& g_{a}^{e}=-0.503 \pm 0.007 \pm 0.016
\end{aligned}
$$

This results can be directly compared (cfr. fig 4) with the more precise measurement of the same quantities done al LEP at $Q^{2} \sim M_{z}^{2}$ using the forward backward asymmetry of the electron and the forward backward asymmetry of the tau polarization. Inside the Standard Model the differences of the two couplings at the two scales are negligible due to a partial cancellation between the changes caused by $\gamma-Z$ transition and the effect of the neutrino charge radius [19]. The agreement is remarkable.

\section{Neutrino nucleon scattering}

A new measurement of $R$, the neutral to charged current interaction rate of neutrino on isoscalar target, has been presented by the CCFR Collaboration[20].

$$
R=\frac{\sigma_{N C}^{\nu}}{\sigma_{C C}^{\nu}}
$$

$R$ is an indirect measurement[21] of the ratio between the $\mathrm{W}$ and the $\mathrm{Z}$ mass with very

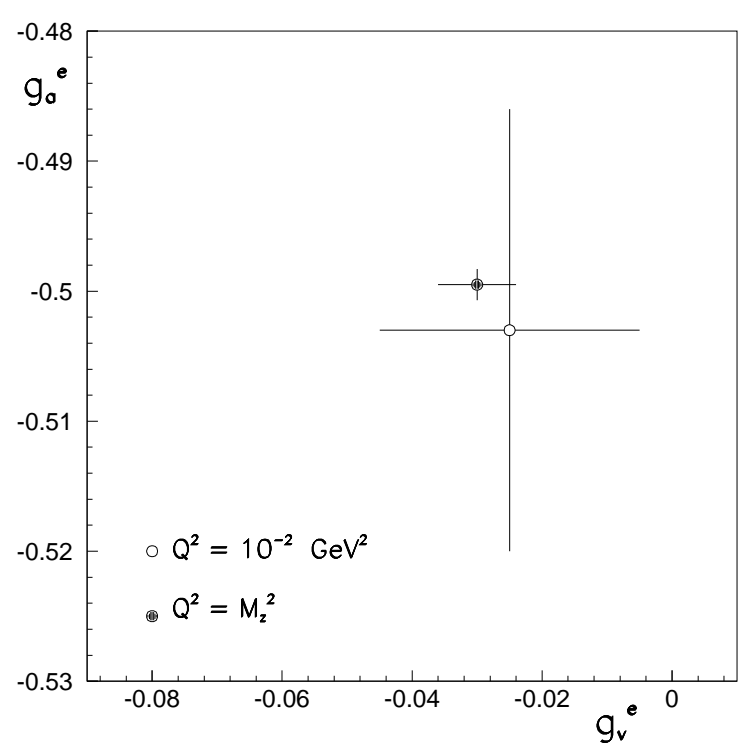

Figure 4. Comparison of results from neutrino electron scattering and LEP.

little dependence on other unknown parameters of the theory

$R=\left(\frac{M_{w}}{M_{z}}\right)^{4} \frac{1}{2} \frac{1-2 \sin ^{2} \theta_{w}+\frac{10}{9} \sin ^{4} \theta_{w}(1+r)}{1-2 \sin ^{2} \theta_{w}+\sin ^{4} \theta_{w}}$

since by numerical accident the dependence of (4) on $\sin ^{2} \theta_{w}$ is very weak. In this formula $\mathrm{r}$ is the ratio between anti-neutrino and neutrino induced charged currents $(r=0.38 \pm 0.01[22$, 23]).

The CCFR collaboration has selected about 140.000 neutral current and 270.000 charged current events using the Fermilab QUAD-TRIPLET neutrino beam. The separation of the events into the two classes is done using the event length measured in the experiment. The main systematic error comes from the model needed for the subtraction of the short charged current events that are misclassified.

The mass ratio is obtained comparing the measured distributions with the result of a complete Monte Carlo simulation:

$$
1-\frac{M_{w}^{2}}{M_{z}^{2}}=0.2242 \pm 0.0029 \pm 0.033 \pm 0.0047
$$

where the first error is statistical, the second 
is due to the experimental systematics and the third to the model used for the interpretation.

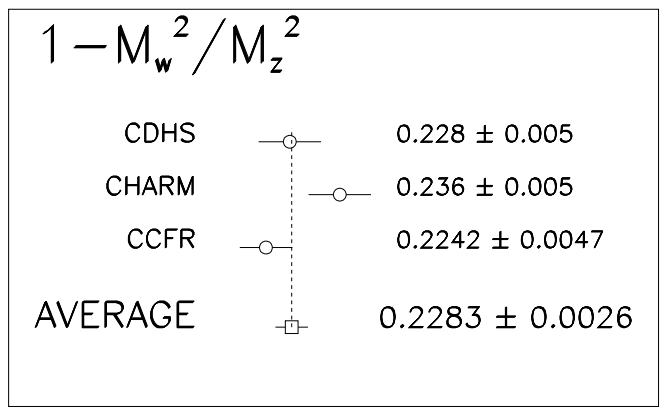

Figure 5. Mass ratio measured by the neutrino nucleon scattering experiments. Only the independent errors are shown and a common systematic error of \pm 0.0047 has to be added to the average. The values of the mass ratio are quoted at different top masses resulting in possible shifts of the central values of 0.002 .

In fig. 5 this result is compared with the measurement done at CERN by CDHS and CHARM[22, 23]. Only the experimental error is shown (including statistics and experimental systematics) since the error due to the model is dominated by the uncertainty on the effective mass of the charm and is very correlated among the three experiments. The correlation is large but not complete since, due to the harder spectrum of the incoming neutrinos, the relative importance of the different terms contributing to the model systematic error is different for CCFR with respect to the two CERN experiments. The combined result of the three experiments is:

$$
1-\frac{M_{w}^{2}}{M_{z}^{2}}=0.2283 \pm 0.0026 \pm 0.0045
$$

and an indirect determination of the $W$ mass

$$
M_{w}=80.10 \pm 0.27 \mathrm{GeV}
$$

is obtained using LEP value $M_{z}=91.187 \pm$ $0.007 \mathrm{GeV}$. This result is consistent with the direct measurement of the $\mathrm{W}$ mass obtained by combining the results of UA2[24] and CDF[25] $\left(M_{w}=80.22 \pm 0.26 \mathrm{GeV}[8]\right)$ and has the same precision.

\section{$\mathrm{Z}$ lineshape and \\ forward backward asymmetries}

The $\mathrm{Z}$ parameters have been measured with high precision at LEP using the data collected in years 1990 and 1991 when various scans of the $Z$ peak were performed. The integrated luminosity delivered by LEP to each experiment was about $25 \mathrm{pb}^{-1}$. The precision on the mass and on the width of the $\mathrm{Z}$ depends crucially on the absolute energy calibration of the machine.

\section{The energy calibration of LEP}

The absolute energy scale of LEP has been calibrated in 1991[26] using the measurements done with a controlled spin-depolarizing resonance on the vertically polarized beam[27] as main tool. Transverse polarization has been observed using a laser polarimeter during eight dedicated experiments with a level between $5 \%$ and $16 \%$ under special conditions, distinct from those of the physics runs and at a single nominal energy setting.

A frequency-controlled radial RF magnetic field makes the electron spin precess away from the vertical axis. A depolarizing resonance occurs when the radial magnetic field oscillates at the spin precession frequency $\omega_{d e p}=2 \pi \nu_{s} f_{r e v}$. The spin tune $\nu_{s}$ is related to the beam energy via

$$
\begin{aligned}
E_{\text {beam }}(G e V) & =\frac{m_{e} c^{2}}{a_{e}} \nu_{s} \\
& =0.4406486\left(N_{s}+\frac{f_{d e p}^{r e s}}{f_{r e v}}\right)
\end{aligned}
$$

where $a_{e}$ is the gyromagnetic anomaly of the electron, and $m_{e}$ is the electron mass. The numerical coefficient in eqn. 5 is known with a precision of $2 \times 10^{-7}$. The integer part of the spin tune at the Z energy is $N_{s}=103$. The intrinsic accuracy of the method is very high and allows a calibration of the average beam energy with an error of $\sim 1 \mathrm{MeV}$. Comparing the spread of six different measurements performed at different times results in an error of 
$\pm 3.7 \mathrm{MeV}$ on the absolute calibration of the center of mass energy of LEP corresponding to a relative accuracy of $4 \times 10^{-5}$.

Other effects have to be taken into account when this calibration is transported to the standard conditions of the physics runs, resulting in a relative accuracy of $5.7 \times 10^{-5}$. The main additional source of error is the change with the temperature of the integrated bending magnetic field seen by the electrons.

The absolute calibration is done at a single nominal energy setting ( $\sim 2 \mathrm{GeV}$ above the $\mathrm{Z}$ mass). The error on the calibration at the other energy settings relative to this special setting depends on the accuracy of the relation between energy and dipole currents, which is determined by magnetic measurements. This error limits the relative accuracy on the measurement of the $\mathrm{Z}$ width to $1.5 \times 10^{-3}$.

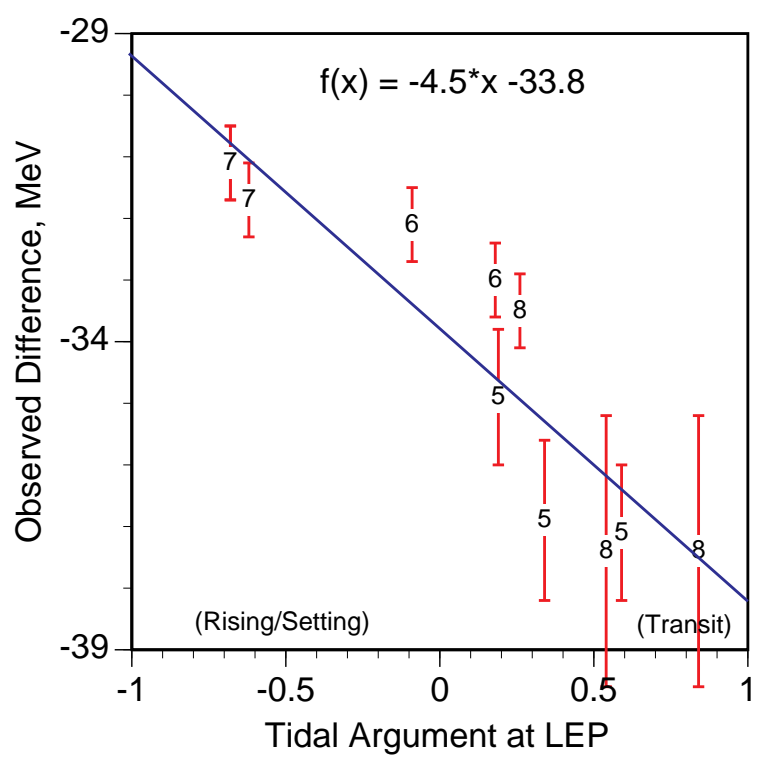

Figure 6. Results of the calibrations done with the resonant depolarization technique as a function of the predicted (normalized) tidal force of the moon and the sun. The numbers at the centre of the error bars indicate the numbering of the calibration experiments.

The error on the $\mathrm{Z}$ parameters induced by the machine calibration is very relevant: the error on the mass is dominated by the calibration error, while the error on the width due to calibration uncertainties is as large as the statistical one. It is important to understand how these errors can be reduced in future measurements. The error on the $\mathrm{Z}$ width can be improved by measuring the local energy scale of LEP performing an absolute calibration at more than one energy setting.

The error on the $\mathrm{Z}$ mass can be improved if the cause for the spread of the beam energies measured with the depolarization technique at different times in ideally identical conditions is understood. A possible explanation is that the radius of LEP changes by a small relative amount $\left(3 \times 10^{-8}\right)$ due to the tidal forces inducing a change of the center of mass energy of $\sim 10 \mathrm{MeV}[28]$. The results of the various calibrations are shown in fig. 6 as a function of the tidal force normalized between -1 and +1 . The data support this hypothesis which however has to be confirmed by a dedicated experiment. If the data are corrected according to the observed correlation, the spread in the calibrations of $\pm 3 \mathrm{MeV}$ is reduced to \pm 1 $\mathrm{MeV}$.

\section{Z lineshape}

The cross sections $e^{+} e^{-} \rightarrow f \bar{f}$ for hadronic $(q \bar{q})$ and leptonic final $\left(\ell^{+} \ell^{-}\right)$states are measured at various energies around $\sqrt{s} \sim$ $M_{z}$. The triggers are highly redundant and their efficiency is essentially $100 \%$ for all channels resulting in negligible systematic errors. The event selections are similar to those performed with 1990 data $[29,30,31,32]$ and better performances of the detectors allow a reduction in the systematic errors. The number of selected events and the systematic errors on the event selections are shown in Table 1 . The uncertainty in the theoretical cross section of the Bhabha scattering used for the luminosity measurement is reduced to the level of $0.3 \%$ by using the BHLUMI[33] computation of the visible cross section which is order $\alpha$ plus Lead- 

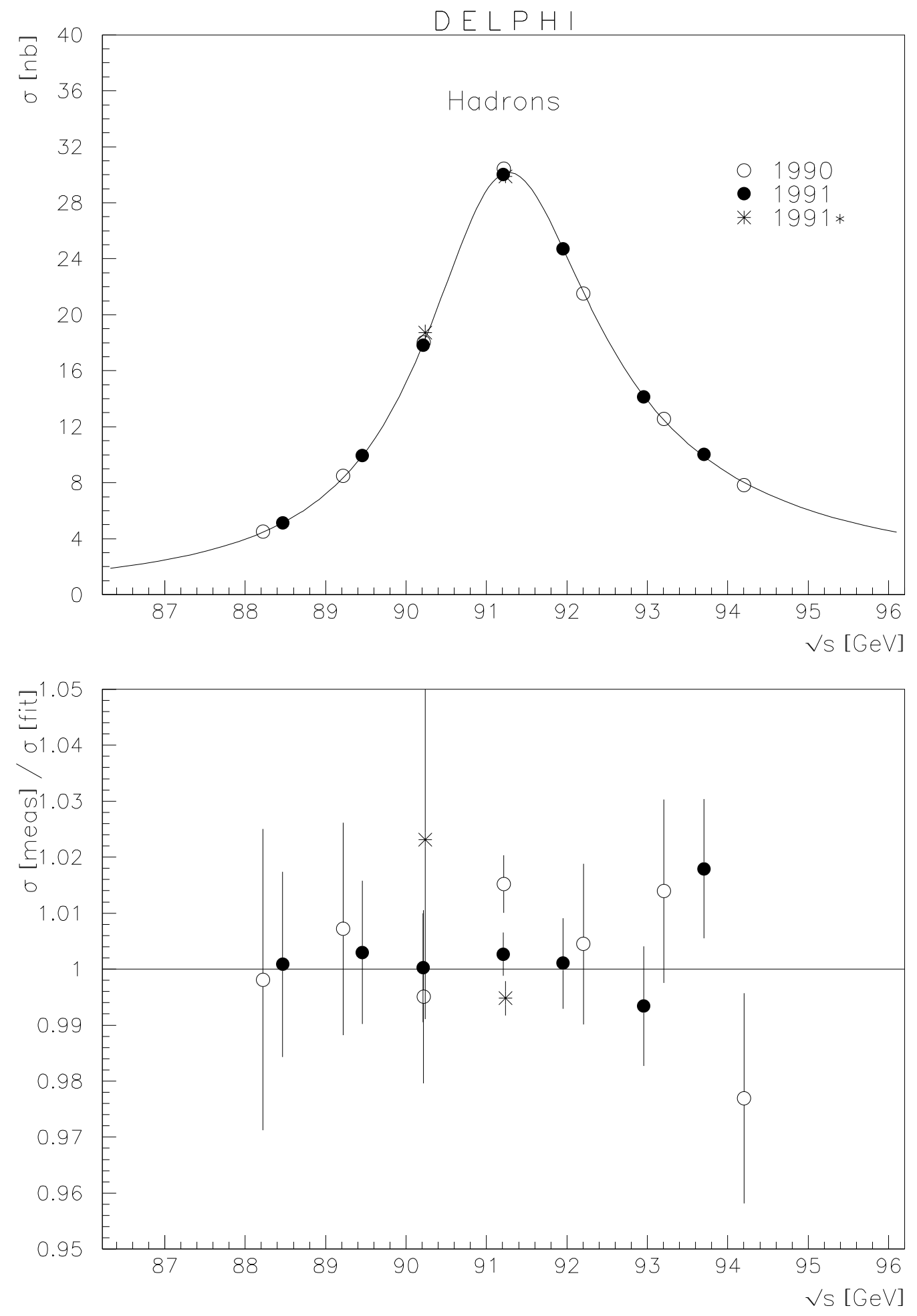

Figure 7. Hadronic cross sections measured by DELPHI. The curve is the result of the fiveparameters fit described in the text 
Table 1. Number of selected events and systematic errors of the event selections. The data sample corresponds to an integrated luminosity of $\sim 25 \mathrm{pb}^{-1}$ luminosity collected in 1990 and 1991 by each experiment.

\begin{tabular}{lccccc}
\hline \hline \multirow{2}{*}{ Number of events } & & ALEPH & DELPHI & L3 & OPAL \\
& $q \bar{q}$ & $452 \mathrm{~K}$ & $368 \mathrm{~K}$ & $425 \mathrm{~K}$ & $454 \mathrm{~K}$ \\
& $\ell^{+} \ell^{-}$ & $53 \mathrm{~K}$ & $35 \mathrm{~K}$ & $41 \mathrm{~K}$ & $54 \mathrm{~K}$ \\
\hline systematic error & $q \bar{q}$ & $0.2 \%$ & $0.3 \%$ & $0.3 \%$ & $0.2 \%$ \\
& $e^{+} e^{-}$ & $0.4 \%$ & $0.5 \%$ & $0.6 \%$ & $0.4 \%$ \\
& $\mu^{+} \mu^{-}$ & $0.5 \%$ & $0.4 \%$ & $1 \%$ & $0.3 \%$ \\
& $\tau^{+} \tau^{-}$ & $0.5 \%$ & $0.9 \%$ & $1 \%$ & $0.8 \%$ \\
\hline experimental systematic & & & & & \\
error on luminosity & & $0.45 \%$ & $0.55 \%$ & $0.85 \%$ & $0.7 \%$ \\
\hline \hline
\end{tabular}

* Opal luminosity systematic error refers to the analysis of 1990 data only

ing Logs resummed. It is complemented with corrections taking into account the high-order terms of the $\gamma-Z$ interference.

The measured cross sections $\sigma^{M}$ are fitted to the expression

$$
\sigma_{f \bar{f}}^{M}(s)=\int H\left(s, s^{\prime}\right) \sigma_{f \bar{f}}^{0}\left(s^{\prime}\right) d s^{\prime}
$$

where $\mathrm{H}$ is the radiator function that takes into account the large $(\sim 30 \%)$ effects of the initial state radiation. $\sigma^{0}$ is the model independent formula of the cross section:

$$
\begin{aligned}
\sigma_{f \bar{f}}^{0}(s)= & \sigma_{f \bar{f}}^{\text {pole }} \frac{s \Gamma_{z}^{2}}{\left(s-M_{z}^{2}\right)^{2}+s^{2} \Gamma_{z}^{2} / M_{z}^{2}} \\
& +\gamma_{\text {exchange }}+\text { Interference } \\
\sigma_{f \bar{f}}^{\text {pole }}= & \frac{12 \pi \Gamma_{e} \Gamma_{f}}{M_{z}^{2} \Gamma_{z}^{2}}
\end{aligned}
$$

Its first term contains the $\mathrm{Z}$ exchange and defines the mass $M_{z}$ and the width $\Gamma_{z}$ of the $\mathrm{Z}$ boson. The photon exchange and the interference terms are small and the Standard Model value is assumed within the fits without any loss in generality at the present level of precision.

\section{Lepton forward-backward asymmetries}

The forward backward asymmetry $A_{F B}^{M}(s)$ is determined through a fit of the angular distribution of the cross section measured at at each center of mass energy:

$$
\frac{d \sigma(s)}{d \cos \theta} \propto 1+\cos ^{2} \theta+\frac{8}{3} A_{F B}^{M}(s) \cos \theta
$$

The measured asymmetry $A_{F B}^{M}(s)$ is fitted to the expression

$$
A_{F B}^{M}(s)=\frac{\int H^{\prime}\left(s, s^{\prime}\right) \sigma_{F B}^{0}\left(s^{\prime}\right) d s^{\prime}}{\int H\left(s, s^{\prime}\right) \sigma^{0}\left(s^{\prime}\right) d s^{\prime}}
$$

where

$$
\sigma_{F B}^{0}=\int_{0}^{1} \frac{d \sigma^{0}}{d \cos \theta} d \cos \theta-\int_{-1}^{0} \frac{d \sigma^{0}}{d \cos \theta} d \cos \theta
$$

The forward backward cross section $\sigma_{F B}^{0}$ depends on the bare asymmetry $A_{F B}^{0}$ :

$$
\sigma_{F B}^{0}\left(M_{z}^{2}\right)=\sigma^{0}\left(M_{z}^{2}\right)(1-\epsilon)\left(A_{F B}^{0}+\delta\right)
$$

where the small term $\epsilon(\sim 0.01)$ is due to the photon exchange and $\delta(\sim 0.002)$ is the contribution of the imaginary part of the propagator corrections. 


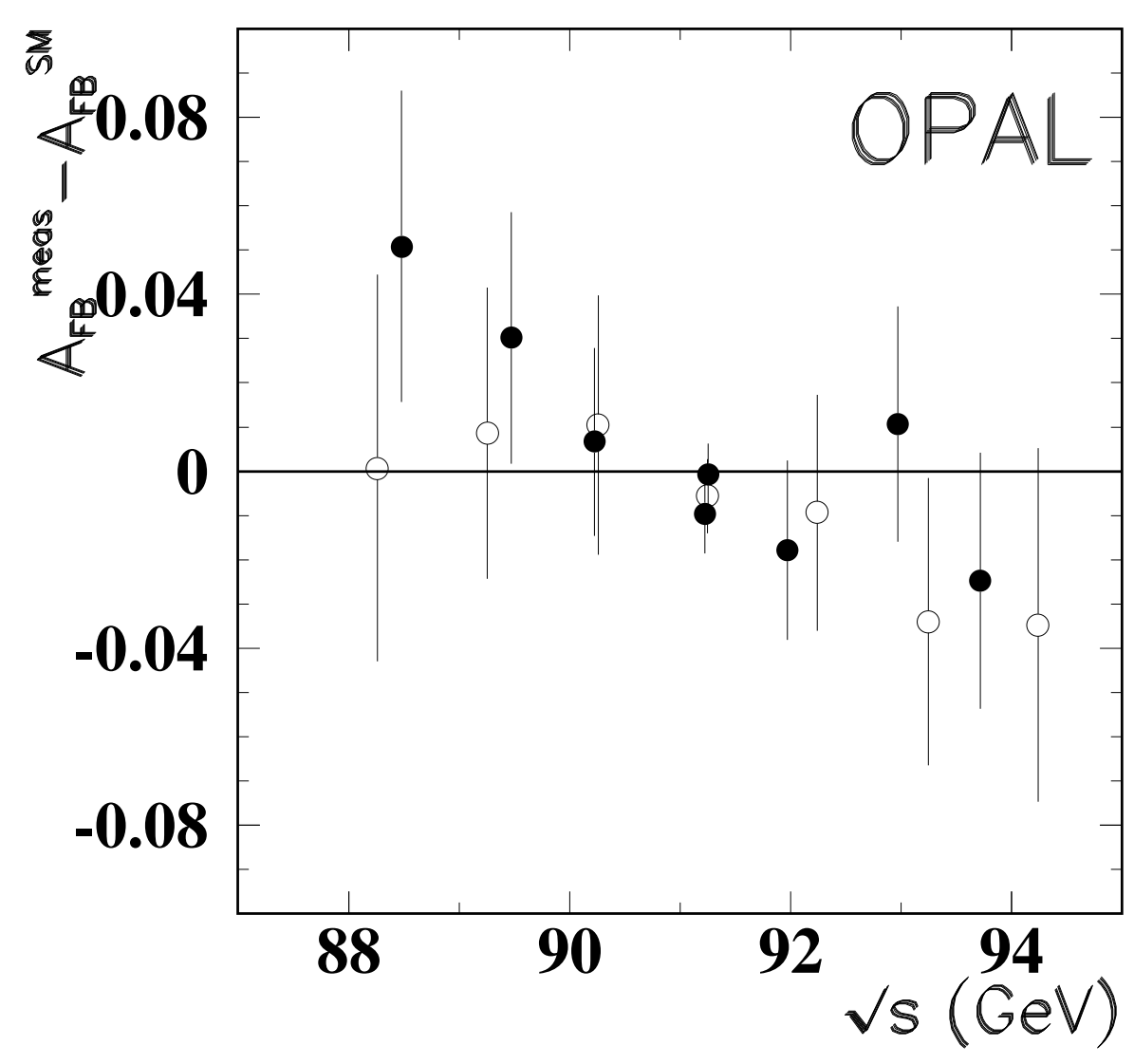

Figure 8. Energy dependence of the lepton forward-backward asymmetry as measured by the OPAL collaboration. The difference between the measured value and the SM prediction for $m_{\text {top }}=150 \mathrm{GeV}$ and $m_{\text {higgs }}=300 \mathrm{GeV}$ is shown.

This bare asymmetry $A_{F B}^{0}$ can be directly interpreted in terms of the ratio between the vector $\left(g_{v}\right)$ and the axial vector $\left(g_{a}\right)$ coupling constants of the neutral current to the fermions:

$$
\begin{aligned}
& \left(A_{F B}^{0}\right)_{x}=\frac{3}{4} A_{e}^{0} A_{x}^{0} \\
& A_{x}^{0}=\frac{2 g_{v}^{x} g_{a}^{x}}{\left(g_{v}^{x}\right)^{2}+\left(g_{a}^{x}\right)^{2}}
\end{aligned}
$$

\section{FIT results}

Two fitting programs are used to extract the lineshape parameters and the bare asymmetries: ZFITTER[35] and MIZA[36]. They give the same result in $2 \%$ of the statistical errors of the fitted parameters[37].
Radiator function H contains the contributions of multiple photon emission and lepton pair production. The error produced on the predicted cross sections by its theoretical uncertainties $\left(\Delta \sigma / \sigma \sim 4 \times 10^{-4}[38]\right)$ is negligible at the present level of precision.

$e^{+} e^{-}$final state contains additional contributions to the cross section from the t-channel diagrams and from the s-t-channel interference. They are taken into account using program ALIBABA[34] resulting in a systematic error smaller then $0.5 \%$.

There are 9 independent parameters to be fitted: the mass and the width of the $Z$, the hadronic peak cross section and, for each lepton species, the ratio $R$ between the hadronic and the leptonic partial widths and the bare 
Table 2. Z lineshape and asymmetry parameters. Lepton universality is not assumed.

\begin{tabular}{c|c|c}
\hline \hline Parameter & Average Value & $\chi^{2}$ \\
\hline$M_{Z}(\mathrm{GeV})$ & $91.187 \pm 0.007$ & 2.3 \\
$\Gamma_{Z}(G e V)$ & $2.492 \pm 0.007$ & 1.5 \\
$\sigma_{q \bar{q}}^{\text {pole }}(n b)$ & $41.16 \pm 0.18$ & 5.3 \\
$R_{e}$ & $20.92 \pm 0.12$ & 3.4 \\
$R_{\mu}$ & $20.79 \pm 0.10$ & 1.2 \\
$R_{\tau}$ & $20.84 \pm 0.13$ & 2.5 \\
$\left(A_{F B}^{0}\right)_{e}$ & $0.0103 \pm 0.0058$ & 1.5 \\
$\left(A_{F B}^{0}\right)_{\mu}$ & $0.0120 \pm 0.0041$ & 4.7 \\
$\left(A_{F B}^{0}\right)_{\tau}$ & $0.0246 \pm 0.0048$ & 1.7 \\
\hline \hline
\end{tabular}

Table 3. Z lineshape and asymmetry parameters. Lepton universality is assumed.

\begin{tabular}{c|c|c}
\hline \hline Parameter & Average Value & $\chi^{2}$ \\
\hline$M_{z}(G e V)$ & $91.187 \pm 0.007$ & 2.3 \\
$\Gamma_{z}(G e V)$ & $2.492 \pm 0.007$ & 1.5 \\
$\sigma_{q \bar{q}}^{\text {pole }}(n b)$ & $41.16 \pm 0.18$ & 5.3 \\
$R_{\ell}$ & $20.85 \pm 0.07$ & 0.5 \\
$\left(A_{F B}^{0}\right)_{\ell}$ & $0.0154 \pm 0.0027$ & 5.5 \\
\hline \hline
\end{tabular}

forward-backward asymmetry. The number of parameters is reduced to 5 when lepton universality is assumed.

The four LEP experiments give consistent results for all fitted quantities. They have been combined to produce the best average values for the $\mathrm{Z}$ lineshape parameters, taking into account errors that are correlated among the experiments. The combination can be done following the procedure described in [39] by taking a simple weighted mean of the fit variables and using the correlation matrix of any of the experiments. The average lineshape and asymmetry parameters from the 9-parameters fit and from the 5-parameters fit are given in Tables 2 and 3. Figure 9 shows the results for the $\mathrm{Z}$ mass. Figure 10 shows the comparison of the results obtained by each experiment

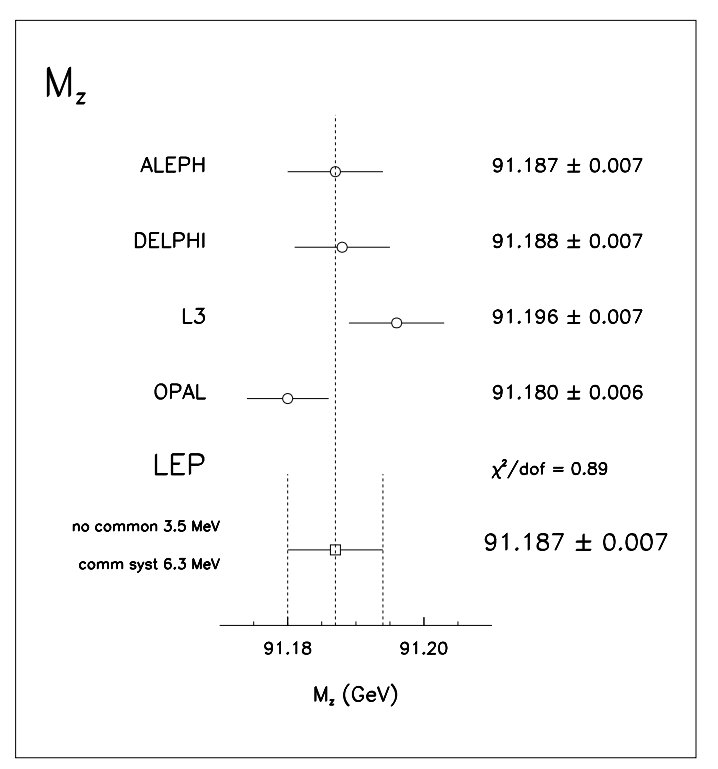

Figure 9. Results for the Z mass.

Table 4. Values of some parameters derived from the result of the 5-parameter fit

\begin{tabular}{c|c}
\hline \hline Parameter & Average Value \\
\hline$\Gamma_{l}(\mathrm{MeV})$ & $83.33 \pm 0.30$ \\
$\Gamma_{\text {had }}(\mathrm{MeV})$ & $1737.1 \pm 6.7$ \\
$\left(g_{v}^{\ell}\right)^{2}$ & $(1.29 \pm 0.23) \times 10^{-3}$ \\
$\left(g_{a}^{\ell}\right)^{2}$ & $0.2494 \pm 0.0009$ \\
$\Gamma_{i n v}(\mathrm{MeV})$ & $504.6 \pm 5.8$ \\
\hline \hline
\end{tabular}

with the standard model prediction. The error on the $Z$ mass is dominated by the uncertainty on the energy calibration of LEP. The error on the width is still statistically limited but the systematic error due to the LEP energy calibration is not negligible. The error on the hadronic peak cross section is dominated in each experiment by the systematic error on the luminosity measurement. Part of this error $(0.3 \%)$ is due to the theory describing the Bhabha scattering and is completely correlated among the four measurements.

The values of other $Z$ parameters and their errors can be derived from the fitted ones. Table 4 shows the values of some derived parameters using the result of the 5-parameters fit. 

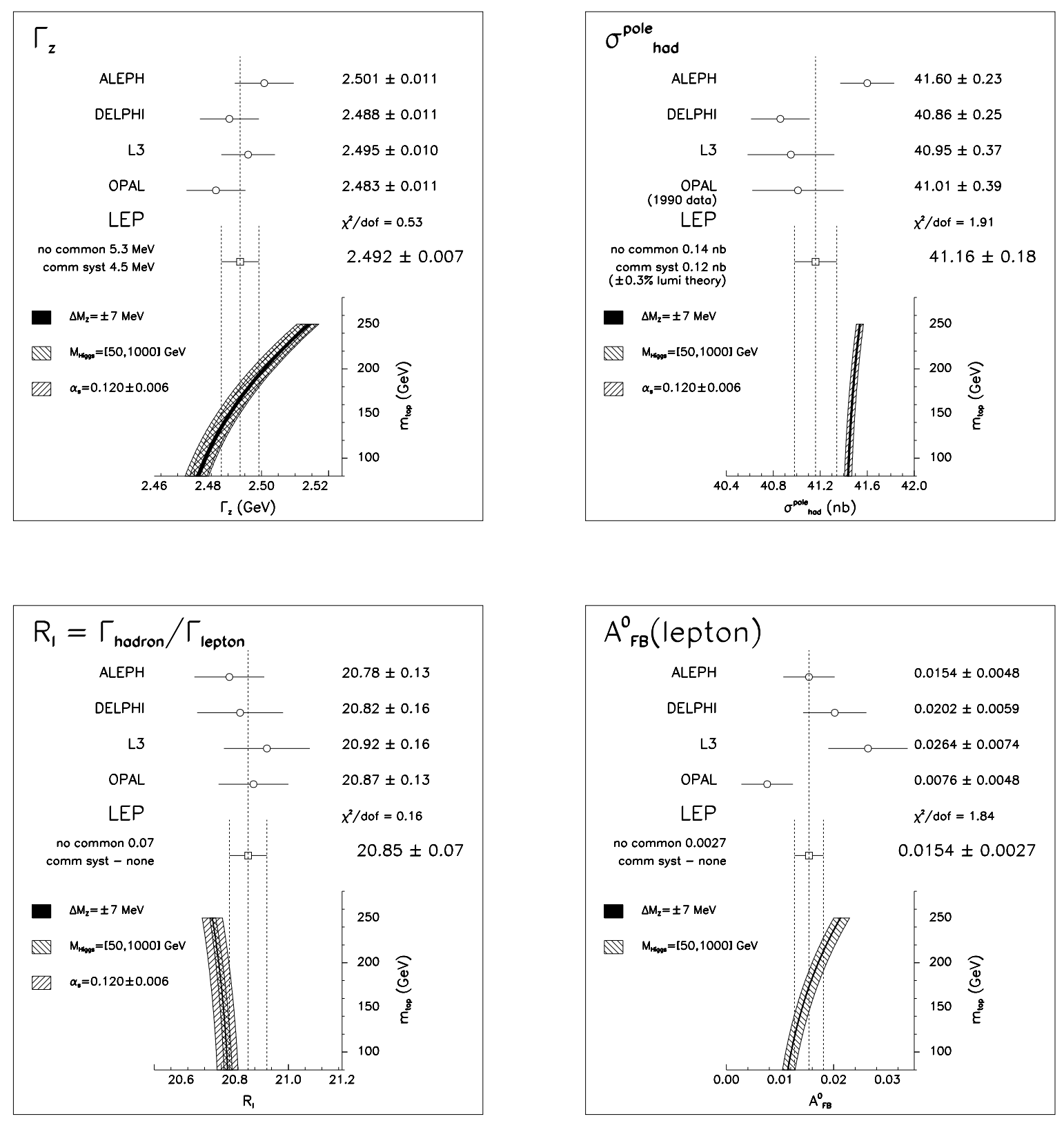

Figure 10 Fit results and Standard Model prediction as a function of $m_{\text {top }}$ for the ranges of $m_{\text {higgs }}$ and $\alpha_{s}$ indicated. 
Tau polarization asymmetry

The angular dependence of the final state longitudinal polarization of the $\tau^{-}$produced in the reaction $e^{+} e^{-} \rightarrow \tau^{+} \tau^{-}$is:

$$
\begin{aligned}
P_{\tau}(\cos \theta) & =\frac{d \sigma_{R}(\cos \theta)-d \sigma_{L}(\cos \theta)}{d \sigma_{R}(\cos \theta)+d \sigma_{L}(\cos \theta)} \\
& =-\frac{A_{\tau}+A_{e} \frac{2 \cos \theta}{1+\cos ^{2} \theta}}{1+A_{\tau} A_{e} \frac{2 \cos ^{2}}{1+\cos ^{2} \theta}}
\end{aligned}
$$

where $\sigma_{R}$ and $\sigma_{L}$ are the cross sections for the production of right-handed and left-handed $\tau^{-}$ and $\theta$ is the angle between the $e^{-}$and the $\tau^{-}$ directions in the center of mass system. At $\sqrt{s} \simeq M_{z}, A_{\tau}\left(A_{e}\right)$ depends on the ratio of the coupling constants of the $\tau(\mathrm{e})$ to the neutral current.

When averaged on all production angles $P_{\tau}$ is a measurement of $A_{\tau}$. Also $A_{e}$ can be extracted when the angular dependence is studied.

The $\tau$ polarization is measured by fitting the momentum distribution of its decay product. Particle identification is applied to select the $\tau$ decay channel. For each channel the measured spectra are fitted with a linear combination of the Montecarlo predicted spectra for the two different helicities, including background and full detector simulation. V-A is assumed in the charged current $\tau$ decay to predict the momentum spectra.

The tau polarization averaged over production angles has been measured at LEP by the four collaborations using the $\tau^{-}$decays into $e^{-} \nu \bar{\nu}, \mu^{-} \nu \bar{\nu}, \pi^{-} \nu$ and $\rho^{-} \nu$. The analysis technique [40] has been improved with respect to the already published result on 1990 data [41, 42, 43], thus reducing systematic errors.

The polarizations obtained by each collaboration in each decay channel are consistent, their averages are shown in fig. 11. Sensitivity is smaller for leptonic decay modes because part of the information is lost in the three body decay with undetectable neutrinos.

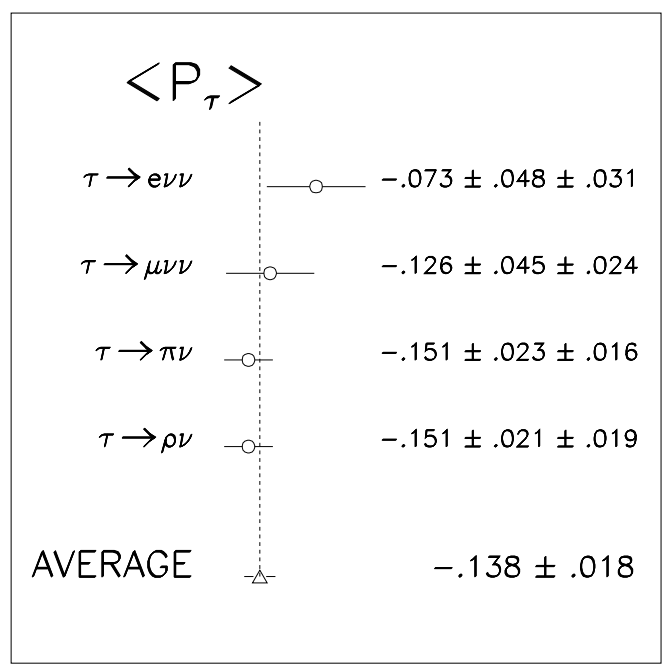

Figure 11. Tau polarization measured using 4 different decay channels. The data shown are averages of the results obtained by the LEP collaborations. The first error is statistical and the second systematic.

The ALEPH collaboration has also studied the angular dependence of the polarization. The polarization averaged over the four different channel is calculated in 9 different $\cos \theta$ bins (cfr. fig. 12). $A_{e}$ is measured from a fit of the angular dependence (cfr. eqn. 10):

$$
A_{e}=0.120 \pm 0.029 \pm 0.010 \text {. }
$$

Some corrections have to be made to convert the measured asymmetry $A_{x}$ to the bare asymmetry $A_{x}^{0}$ (cfr. eqn. 9):

$$
\begin{array}{rlc}
A_{\tau}^{0}=A_{\tau} & *(1-0.002) & \sqrt{(s) \neq M_{z}} \\
& *(1+0.017) & \text { QED initial state } \\
& *(1+0.005) & \gamma_{\text {exch }}+\text { Interf }
\end{array}
$$

The effect of the final state radiation is taken into account in the fit procedure. The same correction factors apply to $A_{e}$.

These correction factors are small. They shift the central value of the measured asymmetry by a small amount compared to the error:

$$
\begin{aligned}
& A_{\tau}^{0}=0.140 \pm 0.018 \\
& A_{e}^{0}=0.122 \pm 0.031
\end{aligned}
$$




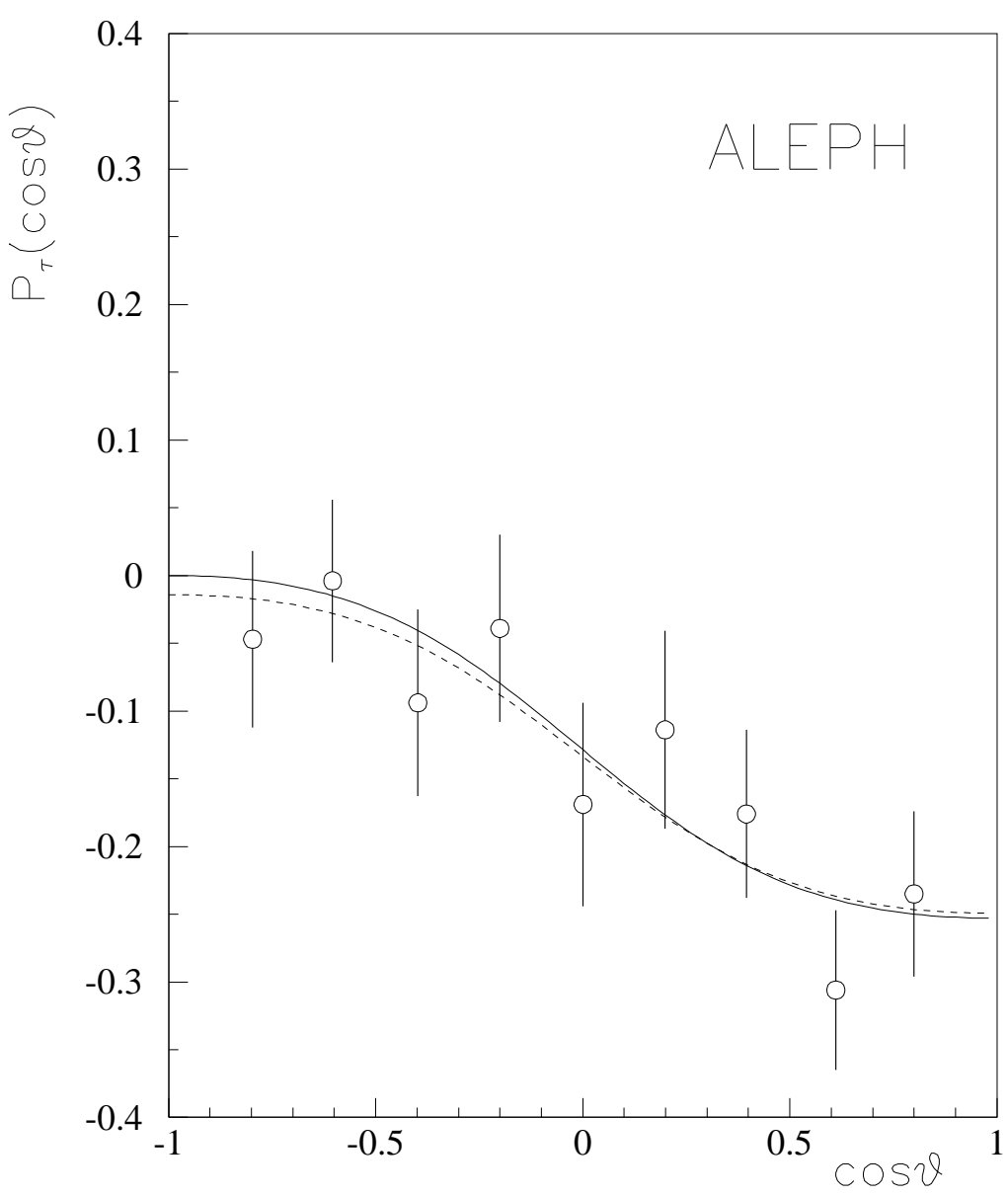

Figure 12. Angular dependence of the $\tau$ polarization measured by the ALEPH collaboration. The full curve is the fit of eqn.10 assuming lepton universality. The dashed line is the fit without the universality assumption.

Neutral current lepton universality

The vector and axial-vector coupling constants of the charged leptons to the neutral current can be calculated by combining the measured width of the $\mathrm{Z}$ into lepton pairs with the asymmetries. The asymmetries measure the ratio between the coupling constants ( $\mathrm{cfr}$ eqn. 9) while the partial widths measure the sum of their squares:

$$
\Gamma_{\ell}=\frac{G_{F} M_{z}^{3}}{6 \sqrt{2} \pi}\left(\left(g_{a}^{\ell}\right)^{2}+\left(g_{v}^{\ell}\right)^{2}\right)\left(1+\frac{3}{4} \frac{\alpha}{\pi}\right) .
$$

The tau polarization is linear in the ratio of the coupling constants thus allowing the determination of the relative sign. The absolute sign is derived from neutrino-electron scattering experiments[18].
Figure 13 shows a comparison among the coupling constants of the three leptons. This comparison is only qualitative since there are correlations between the errors on the coupling constants of different leptons which cannot be shown in the plot.

The coupling constants are equal inside the errors, as is expected from universality. They also agree with the standard model prediction.

The error of $g_{v}^{e}$ and $g_{v}^{\tau}$ is smaller than the error of $g_{v}^{\mu}$ because of the tau polarization constraint.

A quantitative test of the universality can be performed by comparing ratios $\mathrm{R}$, measured for the three lepton species in the 9- 


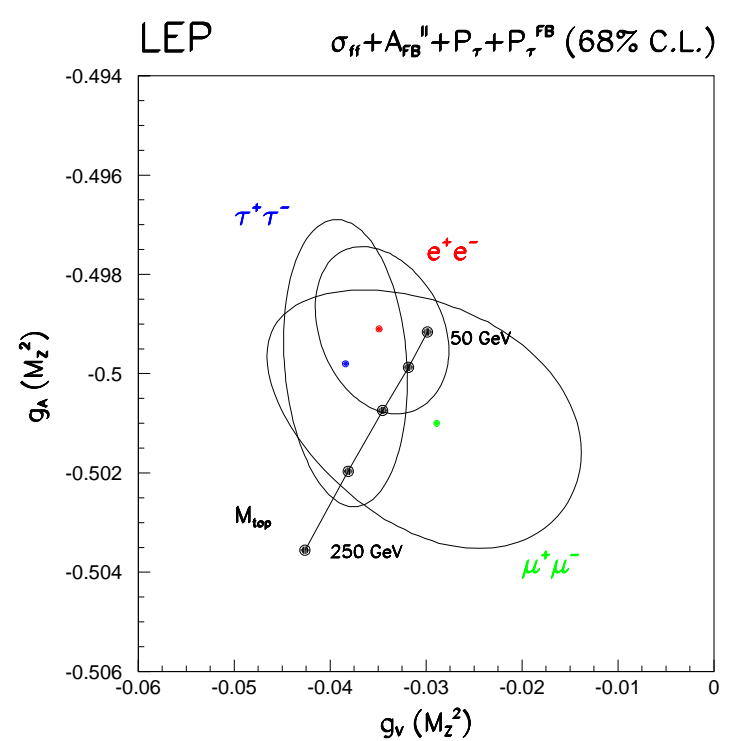

Figure 13. Comparison among the vector and axial vector coupling constants of the neutral current to the leptons.

parameter fit (cfr. tab. 2):

$$
\frac{R_{e}}{R_{\mu}}=\frac{\left(g_{a}^{e}\right)^{2}+\left(g_{v}^{e}\right)^{2}}{\left(g_{a}^{\mu}\right)^{2}+\left(g_{v}^{\mu}\right)^{2}} \simeq\left(\frac{g_{a}^{e}}{g_{a}^{\mu}}\right)^{2} .
$$

This ratio tests the equality of the axial-vector couplings since $g_{v} \ll g_{a}$.

$$
\begin{aligned}
& \frac{g_{a}^{e}}{g_{a}^{\mu}}=1.003 \pm 0.003 \\
& \frac{g_{a}^{\tau}}{g_{a}^{\mu}}=1.001 \pm 0.004
\end{aligned}
$$

\section{$b \bar{b}$ forward backward asymmetry}

The forward backward asymmetry of the $b$ quark produced in the $\mathrm{Z}$ decays at LEP has been measured by tagging the $b \bar{b}$ production with the presence of a prompt lepton with high $p_{T}$ with respect to the parent jet direction. The thrust axis is taken to define the quark direction and the sign of the charge $Q_{\ell}$ of the lepton to distinguish $\mathrm{b}$ from $\bar{b}$.

The raw asymmetry $A_{M}$ is measured by fitting the angular distribution

$$
\begin{gathered}
\frac{d \sigma}{d \cos \theta_{b}} \propto 1+\cos ^{2} \theta_{b}+\frac{8}{3} A_{M} \cos \theta_{b} \\
\cos \theta_{b}=-Q_{\ell} \cos \theta_{\text {THRUST }}
\end{gathered}
$$

Due to the mixing in the $B^{0}-\bar{B}^{0}$ system, the observed b-quark asymmetry is smaller than the actual asymmetry by a factor (1$\left.2 \chi_{B}\right)$, where $\chi_{B}$ is the probability that a hadron containing b-quark has oscillated into a hadron containing $\bar{b}$-quark at the time of its decay.

The high $p_{T}$ lepton sample contains also non-prompt leptons from other sources: cascade decay of the $\mathrm{b}(b \rightarrow c \rightarrow \ell, b \rightarrow \bar{c} \rightarrow \ell)$, charm decay $(c \rightarrow \ell)$ and background. The relation between the true $\left(A_{F B}^{b}\right)$ asymmetry and the measured $\left(A_{M}\right)$ one is:

$$
\begin{aligned}
A_{M}= & \left(1-2 \chi_{B}\right) A_{F B}^{b}\left[\eta_{b \rightarrow \ell}-\eta_{b \rightarrow c \rightarrow \ell}+\eta_{b \rightarrow \bar{c} \rightarrow \ell}\right] \\
& -A_{F B}^{c} \eta_{c \rightarrow \ell}+A_{B K G} \eta_{B K G} .
\end{aligned}
$$

The fractions $\eta$ of each source of leptons are determined via Monte Carlo (cfr fig. 15). The sensitivity of the measurement depends crucially on the purity of the sample $\left(\eta_{b \rightarrow \ell}\right)$.

Fig. 14 shows the new results presented by the LEP collaborations[44] which improve the analysis already performed with lower statistics[45, 46, 47, 48].

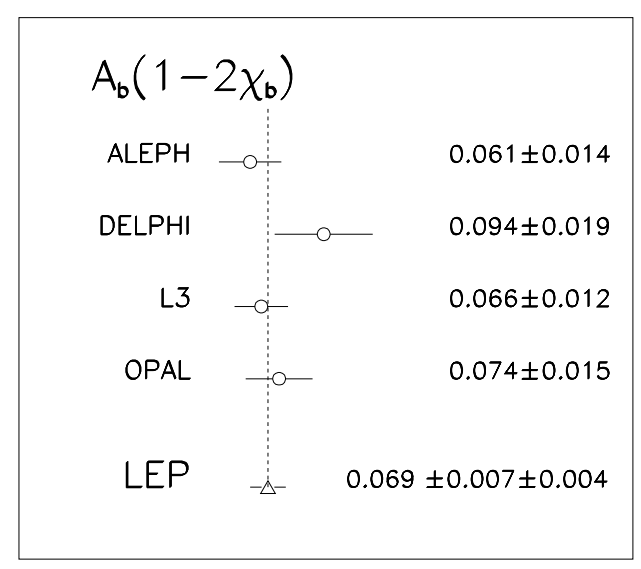

Figure 14. Results for the $b \bar{b}$ asymmetry.

The average shown in fig 14 is done by taking into account common systematic errors. They are due to assumptions on the $\mathrm{B}$ decay modes and on fragmentations functions common to the four experiments[44]. 


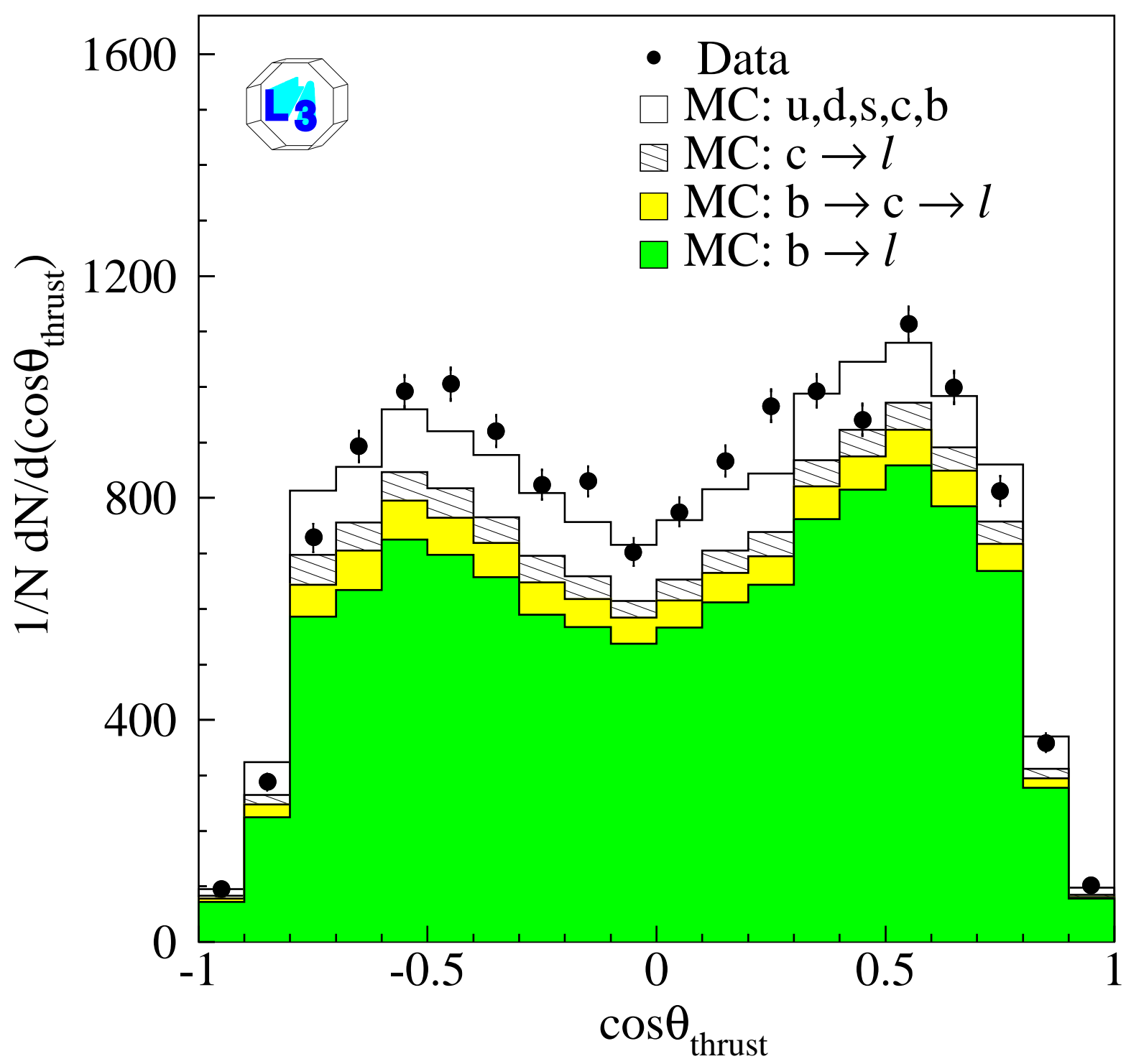

Figure 15. Angular distribution of the thrust axis for events containing a high $p_{T}$ lepton (>1 Gev) measured by the L3 collaboration. The contributions of the various sources are indicated.

Asymmetry $A_{F B}^{b}$ is derived using the Lep average value of $\chi_{B}=0.126 \pm 0.012$ :

$$
A_{F B}^{b}=0.093 \pm 0.010 \pm 0.006
$$

where the first error contains the statistical effects and the uncorrelated systematics and the second contains the correlated systematics including the error on $\chi_{B}$.

Some corrections have to be made to convert asymmetry $A_{F B}^{b}$ to the bare asymmetry $\left(A_{F B}^{0}\right)_{b}$ (cfr. eqn. 9):

$$
\begin{array}{rlc}
\left(A_{F B}^{0}\right)_{b}=A_{F B}^{b} & *(1-0.007) & \sqrt{(s) \neq M_{z}} \\
& *(1+0.040) & \text { QED initial state } \\
& *(1+0.023) & \text { QCD final state } \\
& *(1+0.0002) & \text { QED final state } \\
& *(1-0.003) & \gamma_{e x c h}+\text { Inter } .
\end{array}
$$

They shift the central value of the measured quantity by $\approx 50 \%$ of its error:

$$
\left(A_{F B}^{0}\right)_{b}=\frac{3}{4} A_{e}^{0} A_{b}^{0}=0.0098 \pm 0.0012 .
$$




\section{Left-right asymmetry}

The annihilation cross-section in $e^{+} e^{-}$collision depends on the helicities of the electron and of the positrons. The left-right asymmetry is defined as:

$$
A_{L R}=\frac{\sigma_{L}-\sigma_{R}}{\sigma_{L}+\sigma_{R}}
$$

where $\sigma_{L}$ and $\sigma_{R}$ are the cross sections for lefthanded and right-handed electrons. It is directly related to the electron asymmetry $A_{e}$ (eqn.9): $A_{L R}=A_{e}$.

The left right asymmetry has been measured at SLC by the SLD collaboration[49] using a polarized electron source. It is a very robust measurement since systematic errors due to acceptance and luminosity determination are cancelled in the cross section ratio.

With a statistics of $10^{3} \mathrm{Z}$ decays and a polarization of $21 \%$, SLD has measured:

$$
A_{L R}=A_{e}=0.02 \pm 0.07 .
$$

This result is not yet competitive with LEP, but the foreseen increase in the statistics $\left(10^{4}\right.$ $\mathrm{Z}$ decays) and expecially in the polarization (75\%)[49] of the electron beam will allow SLD to perform a precise measurement of the electron asymmetry.

The effective sinus: $\sin ^{2} \theta_{W}^{e f f}$

The effective sinus is defined in terms of the ratio between the vector and axial vector couplings of the lepton to the neutral current:

$$
\frac{g_{v}^{\ell}}{g_{a}^{\ell}}=1-4 \sin ^{2} \theta_{W}^{e f f}
$$

Assuming lepton universality, the measurements of the leptonic forward backward asymmetry and of $A_{e}^{0}$ and $A_{\tau}^{0}$ from the $\tau$ polarization can be directly converted into $\sin ^{2} \theta_{W}^{e f f}$ using eqn. 9 and 12 .

The $b \bar{b}$ forward backward asymmetry can also be converted into $\sin ^{2} \theta_{W}^{e f f}$ assuming the
Standard Model to evaluate $A_{b}^{0}$. This is a quite safe assumption since in the Standard Model $A_{b}$ is almost independent from $\sin ^{2} \theta_{W}^{e f f}[50]$.

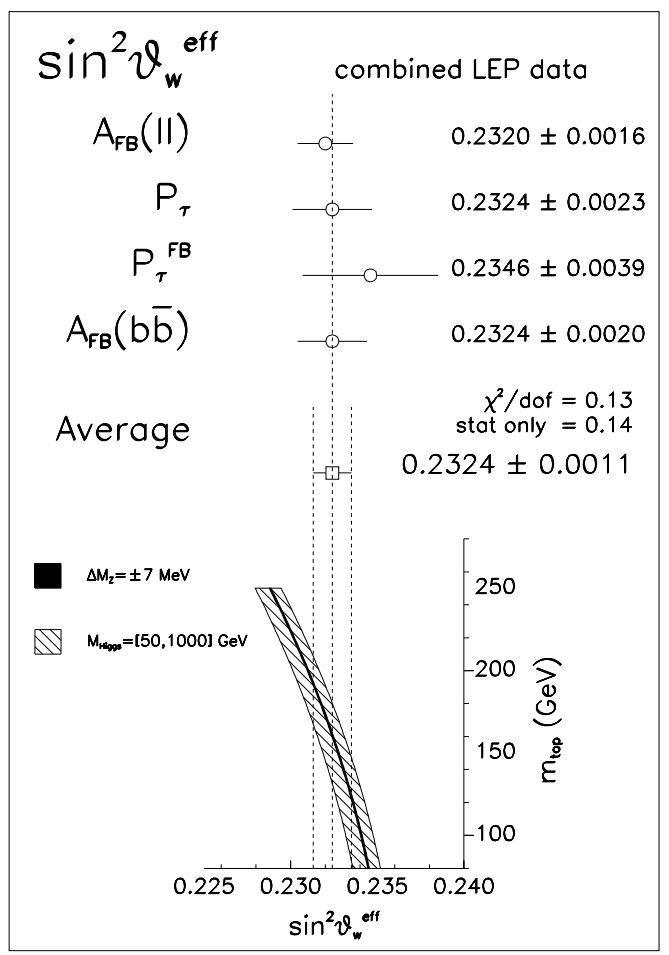

Figure 16. Comparison among different determinations of the effective sinus.

Fig. 16 shows the comparison among the different determinations of $\sin ^{2} \theta_{W}^{e f f}$. They are all very consistent among each other and also with the Standard Model prediction. The average is:

$$
\sin ^{2} \theta_{W}^{e f f}=0.2324 \pm 0.0011 .
$$

Using eqn. 12 this result can be combined with the value of $g_{a}^{\ell}$ from the lineshape fit (cfr. tab. 4) to obtain a more precise evaluation of $g_{v}^{\ell}$ :

$$
\begin{aligned}
& g_{a}^{\ell}=-0.4994 \pm 0.0009 \\
& g_{v}^{\ell}=-0.0351 \pm 0.0022
\end{aligned}
$$

The various measurements shown so far (cfr figs. 10 and 16) are all consistent with the Standard Model for a given range of the top mass and each of them can be used to constrain its value. 


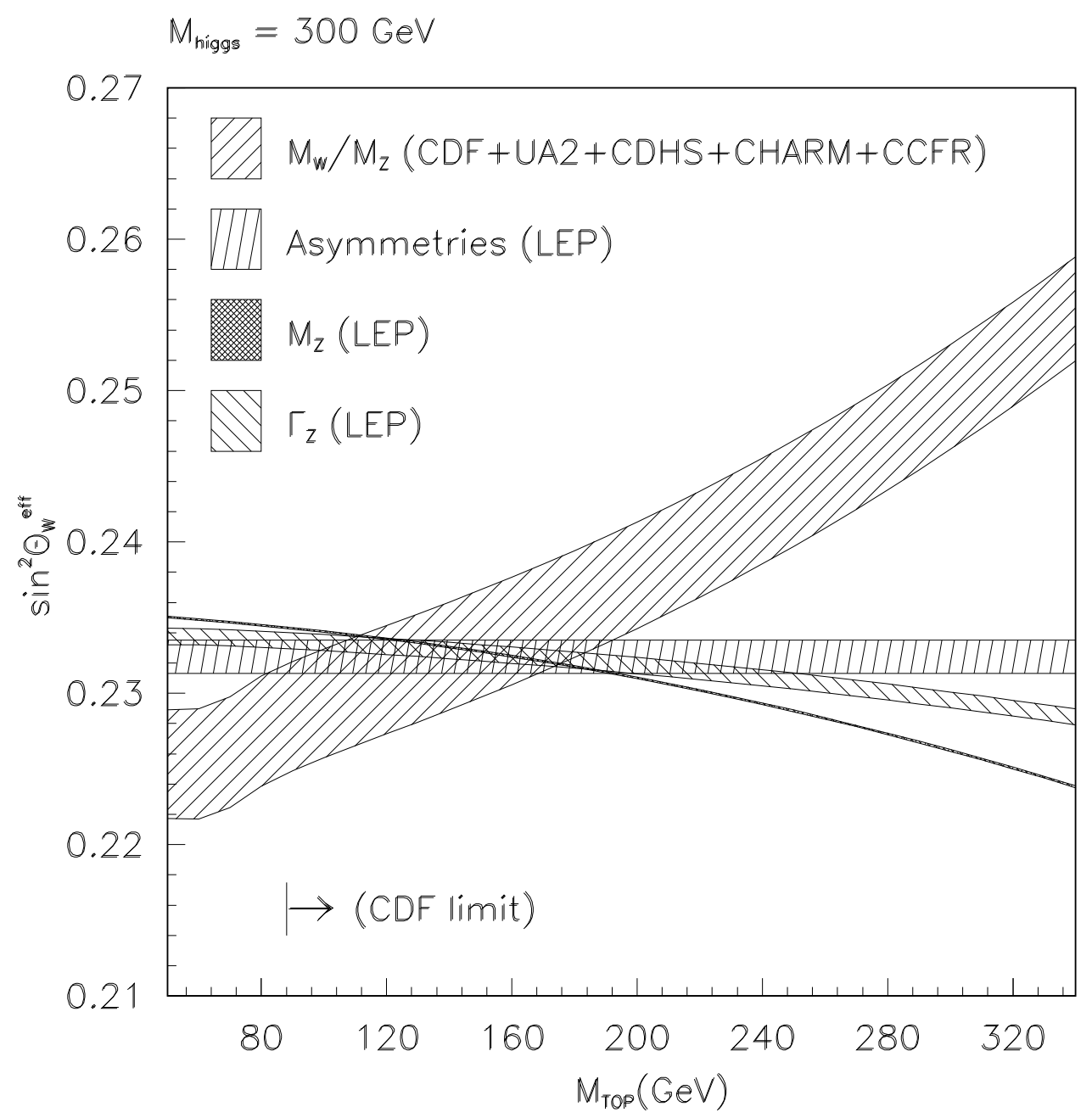

Figure 17. Constraints $\sin ^{2} \theta_{W}^{e f f}$ versus $m_{\text {top }}$ from different measurements corresponding to $1 \sigma$ limits. $m_{\text {higgs }}=300 \mathrm{GeV}$ is assumed.

Fig. 17 shows the consistency among the different constraints: for any value of the top mass and for a fixed value of the Higgs mass (which here is assumed to be $300 \mathrm{GeV}$ ) each experimental measurement can be used to predict $\sin ^{2} \theta_{W}^{e f f}$ within the framework of the Standard Model. The error on the prediction reflects the experimental error on the chosen quantity since all other input parameters have negligible relative error $\left(\alpha, G_{F}\right)$ or are assumed $\left(m_{\text {top }}, m_{\text {higgs }}\right)$. The prediction with its error can be plotted as a function of $m_{\text {top }}$ resulting in an allowed (68\%) region in the plane $\sin ^{2} \theta_{W}^{e f f}$ vs $m_{\text {top }}$.

Four different bands are shown in fig 17 . They are calculated using the measurements of $M_{z}, \Gamma_{z}{ }^{2}$, the asymmetries and mass ratio $M_{w} / M_{z}$. The band produced by the asymmetries is horizontal since they are a direct measurement of $\sin ^{2} \theta_{W}^{e f f}$. The mass ratio value is obtained by combining the results from the neutrino nucleon scattering with those from

\footnotetext{
${ }^{2}$ The strong coupling constant $\alpha_{s}$ is needed to predict $\sin ^{2} \theta_{W}^{e f f}$ from $\Gamma_{z}$. The value $\alpha_{s}=0.120 \pm 0.006$ from LEP event shape is used[51] and the uncertainty induced on the prediction by its error is included in the width of the band.
} 
the direct measurement of the $W$ mass and the LEP value of $M_{z}$. The correlation between the constraint produced by the mass ratio and the very thigh one produced by $M_{z}$ is negligible since the relative error on $M_{z}$ is very small compared to those on the other quantities.

All data are consistent with a top mass within the range $120-180 \mathrm{GeV}$.

\section{Minimal Standard Model fits}

The top mass can be determined by fitting the Standard Model to the various electroweak quantities that are sensitive to it through radiative corrections. In this fit the unknown mass of the Higgs boson is fixed to $300 \mathrm{GeV}$ and the change in the results induced by a change in the Higgs mass from 60 to $1000 \mathrm{GeV}$ is quoted as an additional error.

Two different data sets are used. The first set $(\mathrm{A})$ contains the LEP measurements : $M_{z}$, $\Gamma_{z}, \sin ^{2} \theta_{W}^{e f f}$ from the asymmetries, $\sigma_{q \bar{q}}^{\text {pole }}$ and $R_{\ell}$. The last two are not sensitive to the top mass and are used to constrain the strong coupling constant $\alpha_{s}$. The second set (B) contains the Lep measurements, the mass ratio $M_{w} / M_{z}$ from neutrino-nucleon scattering, $M_{w}$ from UA2[24] and CDF[25], $g_{a}^{e}$ and $g_{v}^{e}$ from the neutrino electron scattering and $Q_{w}$ measured in the parity violation experiment in Cesium[52].

Two different fits are performed. In the first fit the value of $\alpha_{s}$ is further constrained using the measurements done at LEP from the hadronic event shape variables[51] $\left(\alpha_{s}=\right.$ $0.120 \pm 0.006)$. In the second this constraint is released. The results of the four fits are shown in table 5 .

The $\chi^{2}$ of the fits is very good, as is anticipated by the consistency of each measurement with the standard model for the same top mass (cfr. fig 17).

The result of fit 1 is equivalent to an indirect determination of the $\mathrm{W}$ mass $\left(M_{w}=\right.$

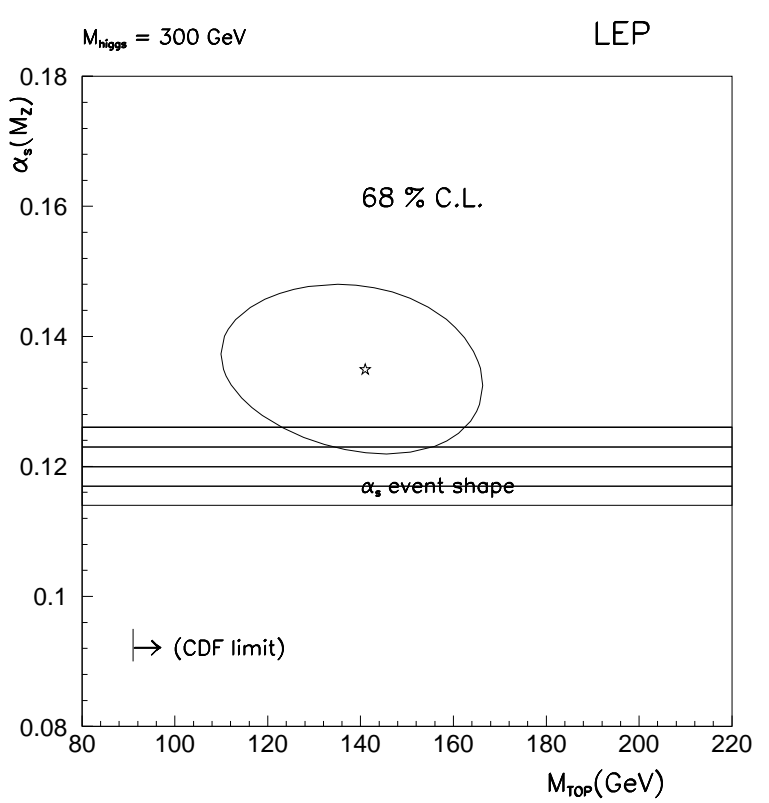

Figure $18 . \alpha_{s}$ vs $m_{\text {top }} 68 \%$ C.L. contour as obtained by fit n.4. The comparison with the $\alpha_{s}$ value obtained from event shape variables is also shown.

$80.17 \pm 0.14 \pm 0.02 \mathrm{GeV}$ ) which is more precise than the direct measurements.

The electroweak data prefer a value of $\alpha_{s}$ slightly higher than the one derived from the event shape variables, but still consistent with it (cfr. fig. 18). The statistical error on $m_{\text {top }}$ is smaller than the uncertainty due to the Higgs mass.

\section{Limits on the Higgs mass}

The limit on the Higgs mass obtained by combining the results of the direct search from the four experiments is [53]:

$$
m_{\text {higgs }}>60 \mathrm{GeV} \text {. }
$$

The effect of the Higgs mass on the radiative correction is small, logarithmic and correlated to the larger effect of the top mass. For this reasons, the electroweak data at the present level of precision cannot constrain the Higgs mass. This is shown in fig 19 where the $\chi^{2}$ of fit 2 is plotted as a function of the top mass for three different values of the Higgs 
Table 5. Results of the standard model fit to top mass and $\alpha_{s}$. The first error is statistical, the second is due to the uncertainty on $m_{\text {higgs }}$.

\begin{tabular}{c|c|c|c|c}
\hline \hline Fit & Data Set & $m_{\text {top }}(\mathrm{GeV})$ & $\alpha_{s}$ & $\chi^{2} /$ d.o.f. \\
\hline 1 & $\mathrm{~A}$ & $147_{-27-22}^{+23+19}$ & constrained & $4.6 / 6$ \\
2 & $\mathrm{~B}$ & $145_{-19-19}^{+17+17}$ & constrained & $7.9 / 14$ \\
3 & $\mathrm{~A}$ & $139_{-30-22}^{+24+19}$ & $0.135 \pm 0.009 \pm 0.002$ & $2.6 / 5$ \\
4 & $\mathrm{~B}$ & $141_{-19-19}^{+17+17}$ & $0.135 \pm 0.009 \pm 0.002$ & $6 / 13$ \\
\hline \hline
\end{tabular}

mass. The data - but in a way that is not significant - prefer lower values of the Higgs mass.

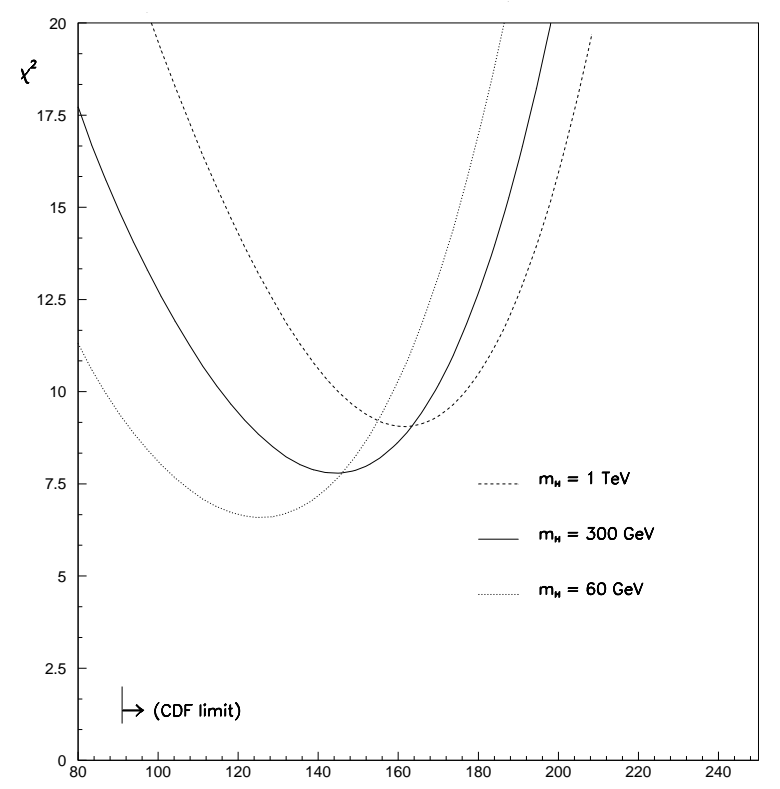

Figure 19. $\chi^{2}$ of fit 2 as function of $m_{\text {top }}$ for three different values of $m_{\text {higgs }}$.

The situation would change if the top were eventually discovered at the Fermilab collider and its mass measured ${ }^{3}$ with an error smaller than the statistical error obtained on $m_{\text {top }}$ in the Standard Model fit. This measurement could be used to further constrain the fit thus increasing the sensitivity to the Higgs mass.

This sensitivity is illustrated in fig. 20,

\footnotetext{
${ }^{3}$ If $m_{\text {top }} \simeq 140 \mathrm{GeV}, \mathrm{CDF}$ should select after cuts $10 e-\mu$ events plus $80 \ell-j e t$ events from $t \bar{t}$ production with $100 \mathrm{pb}^{-1}$ of integrated luminosity[54].
}

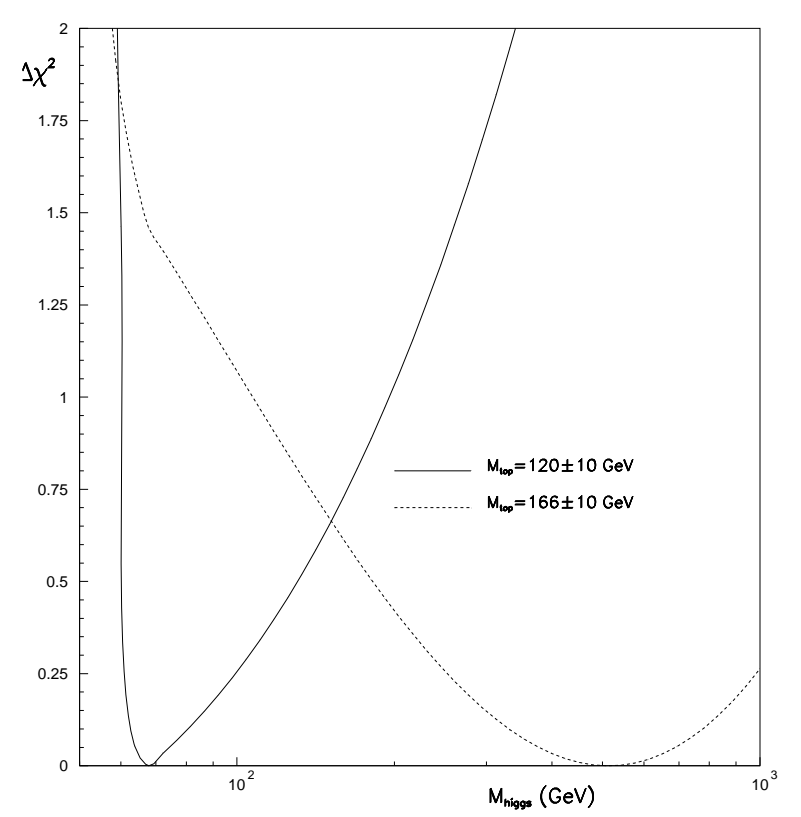

Figure 20. $\chi^{2}$ variation of fit 2 as function of $m_{\text {higgs }}$ for two values of the top mass

where the $\chi^{2}$ variation of fit 2 is shown as a function of the Higgs mass when a constraint on $m_{\text {top }}$ is added to the input data.

The sharp rise of the $\chi^{2}$ for $m_{\text {higgs }}<60$ $\mathrm{GeV}$ is due to the limit set by the direct search. The error on $m_{\text {higgs }}$ increases with $m_{\text {higgs }}$ since its effect on the radiative corrections is logarithmic. Moreover the top and Higgs masses are positively correlated in the fit: a light top mass implies a light Higgs mass.

If the top mass is light $(\simeq 120 \mathrm{GeV})$ the fit prefers a light Higgs mass and could exclude to $1 \sigma$ Higgs masses larger than 200-300 $\mathrm{GeV}$. If the top mass is heavy $(\simeq 160 \mathrm{GeV})$ the 


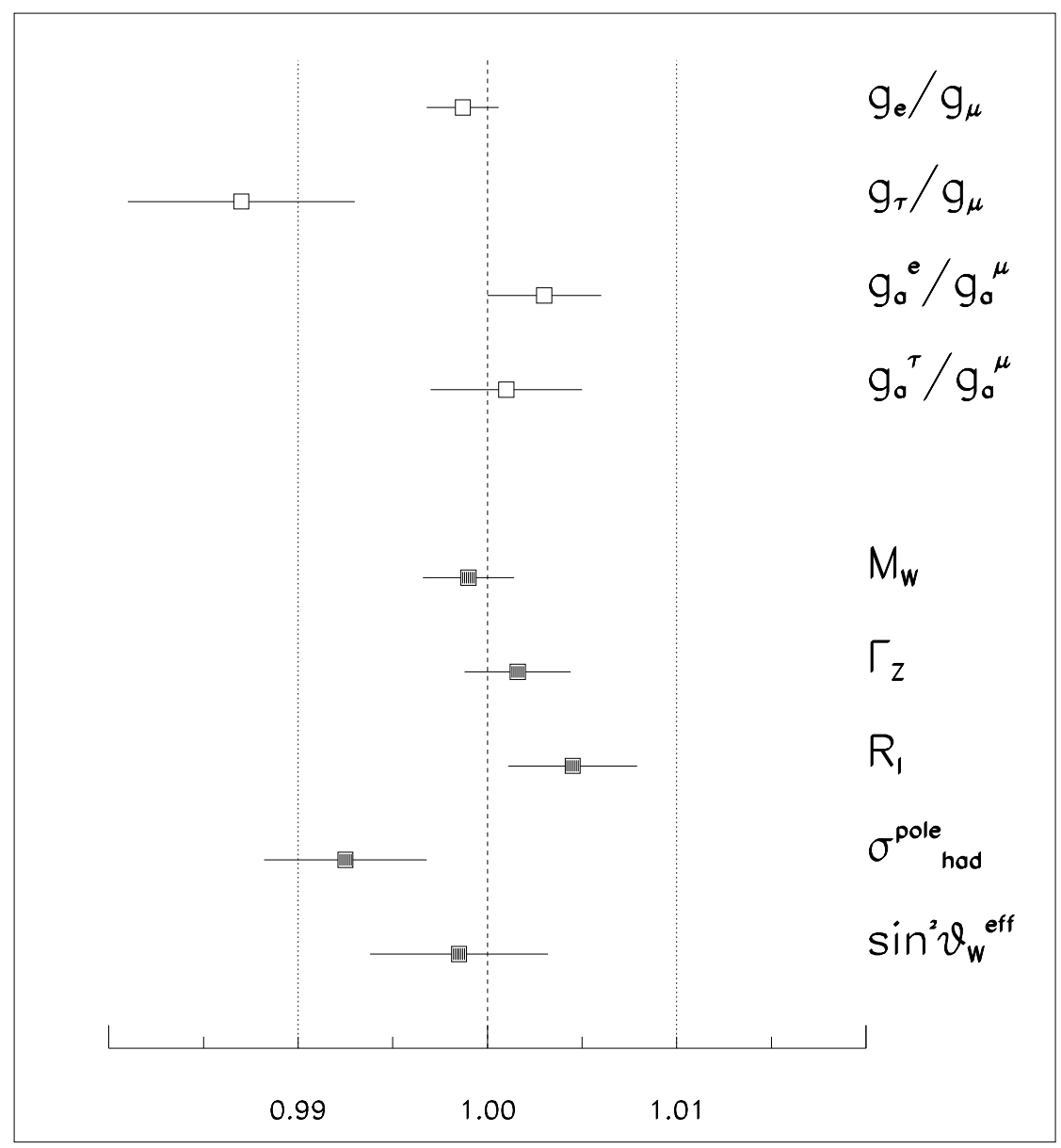

Figure 21. The first four quantities are the ratios of the coupling constants of the leptons which are expected to be equal in the Standard Model. The last five are the ratios between measurements and predictions of the Minimal Standard Model calculated assuming the measured value of the $\mathrm{Z}$ mass, $m_{\text {top }}=150 \mathrm{GeV}, m_{\text {higgs }}=300 \mathrm{GeV}$ and $\alpha_{s}=0.12$. Only experimental errors are shown.

Higgs mass is also heavy and its error would be larger: in this scenario no upper limit on the Higgs mass could be derived from the fit of the electroweak data with the present level of precision even if the top were discovered and its mass measured.

\section{Conclusions}

The precise results obtained by the many experiments testing the Minimal Standard Model in various sectors are all in agreement. This is illustrated in fig. 21, where the ratio between measurements and predictions is shown for various observables. The conclusion is therefore that all data can still be consistently interpreted within the electroweak theory in its minimal form. This is a big success of the theory since the experimental precision on many observables is higher than $0.5 \%$.

Table 6 . Present and future errors on some electroweak observables.

\begin{tabular}{c|c|c|c}
\hline \hline & Now & End '93 & \\
\hline$\Delta M_{W}(\mathrm{MeV})$ & 190 & 100 & CDF + D0 \\
$\Delta \Gamma_{z}(\mathrm{MeV})$ & 7 & 3 & LEP \\
$\Delta \sin ^{2} \theta_{W}^{e f f}$ & 0.0011 & 0.0004 & LEP + SLC \\
\hline \hline
\end{tabular}

The slight discrepancies shown in fig 21 are 
not statistically significant and call for more data to further improve the accuracy of the Precision Tests of the Electroweak interaction.

LEP, SLC and the Fermilab collider are now running and the various experiments are increasing their statistics. In two years the experimental precision on some crucial quantities (as those shown in fig 17) is expected to improve (cfr. table 6) thus putting the electroweak theory to a stronger test. If CDF and D0 find the top and measure its mass with an error $\simeq 5 \mathrm{GeV}$, there is a possibility to be sensitive to the mass of the Higgs boson.

\section{Acknowledgements}

It is a pleasure to thank M. Martinez for the enlightening discussions and for the programs for the Standard Model fits. I am grateful to A. Blondel, J. Lefrançois, R. Tanaka and F. Ragusa for the invaluable help given in preparing this review and to many other colleagues for useful discussions and for the help in providing me with the documentation, in particular: G. Altarelli, J-E. Augustin, S. Bethke, T. Bolton, R. Clare, P. Drell, J. Ellis, F. Fidecaro, M. Franklin, S. Ganguli, S. Gentile, K. Kumar, M. McCubbin, H. Meinhard, J. Mildenberger, C. Moisian, T. Mori, G. Myatt, A. Olshevski, J. Panmann, M. Pohl, G. Quast, M. Roney, P. Rowson, M. Sasaki, D. Schaile, V. Sharma, W. Smith, B. Spaan, D. Treille, W. Toki and T. Wyatt.

\section{REFERENCES}

1. W.J. Marciano, $\tau$ decay puzzle, Phys. Rev. D45, R721, (1992).

2. W.J. Marciano, private communication (1990)

3. D.I. Britton et al, Measurement of the $\pi^{+} \rightarrow e^{+} \nu$ Branching ratio, Phys. Rew. Lett. 68,3000,(1992).
4. G. Czapek et al, Branching ratio of the Rare Pion Decay into positron and neutrino, LHEP-Preprint BUHE-92-1(1992).

5. W. Marciano and A. Sirlin, Electroweak radiative corrections to $\tau$ decay, Phys. Rev. Lett. 61,1815,(1988).

6. M. Danilov, Heavy Flavour Physics, in Proceedings of the HEP91 Conference, 1991, p. 333.

7. J.Z. Bai et al, Measurement of the mass of the $\tau$ lepton, SLAC-PUB 5870 and BEPC-EP-92-01, (1992), and F. Porter, Talk given at this conference in PA-1.

8. Particle Data Group, K. Hikasa et al, Review of particle properties, Phys. Rev. D, 45, 1 (1992).

9. ARGUS Collaboration, A measurement of the tau mass, DESY 9D-86, (1992) and A. Golutvin, Talk given at this conference in PA-1.

10. CLEO Collaboration, K. Gan, Tau physics at CLEO, Talk given at this conference in PA-1.

11. ALEPH Collaboration, D. Decamp et al, Measurement of the $\tau$ lifetime, Phys. Lett. B297, 411, (1992).

12. DELPHI Collaboration, P. Abreu et al, A measurement of the lifetime of the $\tau$ lepton, Phys. Lett. B267, 422, (1991).

13. L3 Collaboration, B. Adeva et al, Decay properties of the $\tau$ lepton measured at the Z resonance, Phys. Lett. B265, 451, (1991).

14. OPAL Collaboration, P.D. Acton et al, Measurement of the $\tau$ lepton lifetime, Phys. Lett. B273, 355, (1991).

15. M. McCubbin, Lifetime and branching ratio of the $\tau$, Talk given at this Conference in $\mathrm{PA}-5$.

16. R. Stroynowski, Tau decays at CLEO, in Proceedings of the HEP91 Conference, 
1991, p. 562.

17. CHARM II Collaboration, A.Staude, NC coupling constants from neutrino electron scattering, talk given at this conference in PA-5.

18. CHARM II Collaboration, P. Vilain et al, Neutral current coupling constants from neutrino and antineutrino electron scattering, Phys. Lett. B281, 159, (1992). A.Staude talk given at this conference in PA-5.

19. V.A. Novikov, L.B. Okun, M.I. Vysotsky, Electroweak radiative corrections and the top mass, CERN TH-6053/91. (1991)

20. CCFR Collaboration, T.Bolton, Results from the CCFR experiment, talk given at this conference in $\mathrm{PA}-5$.

21. R.G. Stuart, The $\bar{M} S$ and the on - shell schemes in the analysis of neutrino scattering experiments, Z. Phys. C34, 445, 1987.

22. CDHS Collaboration, A. Blondel et al, Electroweak parameters from a high statistics neutrino nucleon scattering experiment, Z. Phys. C, 45, 361, (1990).

23. CHARM Collaboration, J.V.Allaby et al, A precise determination of the electroweak mixing angle from semileptonic neutrino scattering, Z. Phys. C 36, 611, (1987).

24. UA2 Collaboration, J. Alitti et al, An improved determination of the ratio of $\mathrm{W}$ and $\mathrm{Z}$ masses at the CERN $p \bar{p}$ collider, Phys. Lett. B276, 354, (1992).

25. CDF Collaboration, F. Abe et al, Measurement of the $\mathrm{W}$ boson mass in 1.8 Tev $p \bar{p}$ collisions, Phys. Rew. D43, 2070, (1991).

26. L. Arnaudon et al, The energy calibration of LEP in 1991, CERN-PPE/92-125 (1992) and G. Quast talk given at this conference in PA5.
27. L. Arnaudon et al, Measurement of LEP beam energy by resonant spin depolarization, CERN-PPE/92-49 (1992).

28. A. Hofmann, Effects modifying the c.m. energy in LEP in Proceedings of the second workshop on LEP performance, CERN SL/92-12, (1992).

29. ALEPH Collaboration, D. Decamp et al, Improved measurement of electroweak parameters from $Z$ decays into fermion pairs, Z. Phys. C53, 1,(1992).

30. DELPHI Collaboration, P. Aarnio et al, Determination of the $Z$ resonance parameters and couplings from its hadronic decays, Nucl. Phys. B367, 511,(1991).

31. L3 Collaboration, B. Adeva et al, Measurement of electroweak parameters from hadronic and leptonic decays of the Z, Z. Phys. C51, 179,(1991).

32. OPAL Collaboration, G. Alexander et al, Measurement of the $\mathrm{Z}$ lineshape parameters and the electroweak couplings of the charged leptons, Z. Phys. C52, 175,(1991).

33. S. Jadach et al, Monte Carlo program BHLUMI 2.01 for Bhabha scattering at low angle with Yenny-Frautshi-Suuna exponentiation, CERN-TH 6230/91, (1991).

34. W. Beenakker et al, Large angle Bhabha scattering, Nucl. Phys. B349, 323, (1991).

35. DUBNA-ZEUTHEN radiative correction group, D. Bardin et al, CERN-TH 6443/92, (1992) and references therein.

36. M. Martinez et al, Model independent fitting of the $\mathrm{Z}$ lineshape, Z. Phys. C49, 645, (1991)

37. T. Riemann, ZFITTER: An analytical program for $e^{+} e^{-} \rightarrow f \bar{f}$. Talk given at this conference in PA-5.

38. S. Jadach et al, Light pair corrections to the $\mathrm{Z}$ lineshape parameters, Phys. Lett. B280, 129, (1992). 
39. The LEP Collaborations, Electroweak parameters of the $Z$ resonance and the Standard Model, Phys. Lett. B199, 224, (1992).

40. K. Kumar, $\tau$ polarization, Talk given at this Conference in PA-5.

41. ALEPH Collaboration, D. Decamp et al, Measurement of the polarization of the $\tau$ lepton produced in Z decays, Phys. Lett. B265, 430, (1991).

42. DELPHI Collaboration, P. Abreu et al, A study of the decays of the $\tau$ leptons produced on the $\mathrm{Z}$ resonance at LEP, CERNPPE 92/60, (1992).

43. OPAL Collaboration, G. Alexander et al, Measurement of the branching ratios and $\tau$ polarization from $\tau$ decays at LEP, Phys. Lett. B266, 201, (1991).

44. T. Wyatt, Forward-backward asymmetries, quarks. Talk given at this Conference in PA-5.

45. ALEPH Collaboration, D. Decamp et al, Measurement of the forward backward asymmetry in $Z \rightarrow b \bar{b}$ and $Z \rightarrow c \bar{c}$, Phys. Lett. B263, 325, (1991).

46. DELPHI Collaboration, P. Abreu et al, A measurement of the $b \bar{b}$ forward backward asymmetry using the semileptonic decay into muons, Phys. Lett. B276, 536, (1992).

47. L3 Collaboration, B. Adeva et al, A measurement of the $Z \rightarrow b \bar{b}$ forward backward asymmetry, Phys. Lett. B252, 713, (1991).

48. OPAL Collaboration, M.Z. Akrawy et al, A study of heavy flavour production using muons in hadronic Z decays, Phys. Lett. B263, 311, (1991).

49. P. Rowson, Polarized electron beam results from SLC, Talk given at this Conference in $\mathrm{PA}-5$.

50. M. Bohm and W. Hollik, Forwardbackward asymmetries, in $Z$ physics at LEP 1, CERN 89-08.
51. S. Bethke, Test of QCD, Talk given at this Conference in PL-4.

52. M.C. Noecker et al, Precision Measurements of Parity Nonconservation in Atomic Cesium: A low-energy test of the electroweak theory, Phys. Rev. Lett. 61, 310, (1988).

53. T. Mori, Searches for the neutral Higgs boson at LEP, Talk given at this Conference in $\mathrm{PA}-13$.

54. J. Huth, Top quark search from CDF, Talk given at this Conference in PA-13.

\section{J. Haissinski, LAL Orsay:}

To make a full use of a very precise measurement of $\sin ^{2} \theta_{W}$ one needs to know with an increased accuracy the running of $\alpha$ between $Q^{2} \simeq 0$ and $Q^{2} \simeq M_{z}^{2}$. This requires better data on the cross section $e^{+} e^{-} \rightarrow$ hadrons at relatively low energies. It would be nice if such measurements could be performed at BEPS in Beijing. 